\title{
Differential ion dehydration energetics explains selectivity in the non-canonical lysosomal $\mathrm{K}^{+}$channel TMEM175
}

SeCheol Oh${ }^{1 *}$, Fabrizio Marinelli2*, Wenchang Zhou $^{2 *}$, Jooyeon Lee ${ }^{3}$, Ho Jeong Choi ${ }^{3}$, Min Kim ${ }^{3}$, José D. Faraldo-Gómez ${ }^{2 \#}$ and Richard K. Hite ${ }^{1 \#}$

$10 \quad{ }^{2}$ Theoretical Molecular Biophysics Laboratory, National Heart, Lung and Blood Institute, 11 National Institutes of Health, Bethesda, Maryland, USA

$12{ }^{3}$ Department of Chemistry, Chungbuk National University, Republic of Korea

13

$14 \quad *$ These authors contributed equally

15 \#Corresponding authors. Email: hiter@mskcc.org, jose.faraldo@nih.gov 


\section{Abstract}

18 Structures of the human lysosomal $\mathrm{K}^{+}$channel TMEM175 in open and closed states revealed a

19 novel architecture lacking the canonical $\mathrm{K}^{+}$selectivity filter motif present in previously known

$20 \mathrm{~K}^{+}$channel structures. A hydrophobic constriction composed of four isoleucine residues was

21 resolved in the pore and proposed to serve as the gate in the closed state, and to confer ion

22 selectivity in the open state. Here, we achieve higher-resolution structures of the open and closed

23 states and employ molecular dynamics simulations to analyze the conducting properties of the

24 putative open state, demonstrating that it is capable of permeating $\mathrm{K}^{+}$ions at the expected rate.

25 Monovalent cations must dehydrate significantly to penetrate the narrow hydrophobic

26 constriction, but ion flow is assisted by a favorable electrostatic field generated by the protein

27 that spans the length of the pore. The balance of these opposing energetic factors explains why

28 permeation is feasible, and why TMEM175 is selective for $\mathrm{K}^{+}$over $\mathrm{Na}^{+}$, despite the absence of

29 the canonical selectivity filter. Accordingly, mutagenesis experiments reveal an exquisite

30 sensitivity of the channel to perturbations that mitigate the constriction. Together, these data

31 reveal a novel mechanism for selective permeation of ions by TMEM175 that is unlike that of

32 other $\mathrm{K}^{+}$channels. 


\section{Introduction}

Transmembrane protein 175 (TMEM175) is an evolutionarily distinct potassium $\left(\mathrm{K}^{+}\right)$ selective cation channel expressed in lysosomes (Cang et al., 2015). Point mutations in

TMEM175 have been identified that are strongly associated with development of Parkinson's

Disease (PD) (Blauwendraat et al., 2019; Iwaki et al., 2019; Jinn et al., 2019; Jinn et al., 2017;

Nalls et al., 2014; Wie et al., 2021). For example, the M393T point mutation is a loss-of-function

mutation associated with the increased likelihood and early onset of PD and the Q65P mutant is a gain-of-function mutation associated with a reduced likelihood of developing of PD (Wie et al., 2021). While the precise mechanisms by which these mutations are associated with PD remain unclear, it is well established that TMEM175 deficiency can lead to numerous defects in the endolysosomal system including dysregulation of lysosomal $\mathrm{pH}$, defects in autophagy and mitophagy and an increased susceptibility to $\alpha$-synuclein cytotoxicity (Jinn et al., 2019; Jinn et al., 2017).

To begin to understand the mechanisms that underlie TMEM175 function in lysosomes, we determined the structure of human TMEM175 by cryo-EM and identified two conformational states (Oh et al., 2020). Consistent with its evolutionarily distinct sequence, the architecture of TMEM175 does not resemble that of any other known ion channel. TMEM175 protomers consist

51 of two homologous repeats of 6 transmembrane (TM) helices, which assemble into a dimer to

52 form the channel. A two-fold symmetric ion conduction pathway runs along the center of the dimer, lined by two copies of TM1 and TM7, the first helices of each repeat. Unlike most cation channels, including canonical $\mathrm{K}^{+}$channels such as KcsA and Shaker, the pore of TMEM175 contains no clusters of hydrophilic side chains or backbone atoms that control selectivity (Doyle 
57 is a narrow hydrophobic constriction formed by the side chains of the conserved Ile46 from TM1 and the conserved Ile271 from TM7, which we termed the isoleucine constriction (Oh et al., 2020). In one of the two cryo-EM structures the isoleucine constriction is too narrow to accommodate $\mathrm{K}^{+}$ions and thus we assigned it as a closed state. In the other structure, however,

61 the isoleucine constriction is sufficiently dilated to accommodate partially hydrated $\mathrm{K}^{+}$ions, and

62 therefore we proposed that this structure corresponds to a conductive state of the channel.

63 Moreover, because ions would need to be partially dehydrated to traverse the pore, we and others

64 proposed that the relative energetics of $\mathrm{K}^{+}$and $\mathrm{Na}^{+}$dehydration through the isoleucine constriction contributes to the ion selectivity of TMEM175 (Lee et al., 2017; Oh et al., 2020). While plausible, this proposal relies on the assumption that the putative open state is indeed conductive; however, assigning functional states to cryo-EM structural snapshots is challenging. Here, we employ high-resolution cryo-EM structure determination, molecular dynamics (MD)

69 simulations and electrophysiological analyses to investigate the mechanisms of ion permeation and selectivity in TMEM175.

\section{Results}

\section{Improved cryo-EM structures of hTMEM175}

Taking advantage of recent advances in cryo-EM image analysis, we reprocessed our published cryo-EM images of TMEM175 in $\mathrm{KCl}$ to achieve higher-resolution (Punjani and Fleet,

76 2021; Punjani et al., 2020; Zivanov et al., 2018). The resolution of the putative open state

77 improved from $2.64 \AA$ to $2.45 \AA$ while the closed state improved from $3.03 \AA$ to $2.61 \AA$ (Figure

78 1-Figure supplement 1 and Table S1). While the improved structures are similar to those

79 previously determined, the higher-resolution reconstructions allowed us to better define the ion 
80 conduction pathways (Figure 1A-D). In both states, this pathway is narrow and largely devoid of

81 polar contacts, particularly near the center due to the presence of several hydrophobic side chains

82 including Ile46, Va150, Leu53, Ile271, Leu275 and Leu278 (Figure 1C-D). In the closed state,

83 the isoleucine constriction formed by Ile46 and Ile271 is clearly too narrow to allow ion

84 permeation (Figure 1D). The side chains of Ile271 point towards the channel axis, narrowing the

85 pore to a minimum radius of $0.2 \AA$ (Figure 1D,E). In the open state, by contrast, conformational

86 changes in the pore-lining helices TM1 and TM7 helices dilate the isoleucine constriction to a

87 minimum radius of $1.6 \AA$, which appears to be sufficient to accommodate a partially dehydrated

$88 \mathrm{~K}^{+}$ion (Figure 1C,E). In its dilated conformation, the isoleucine constriction adopts a nearly

89 four-fold symmetric configuration with the side chains of both Ile46 and Ile271 adopting the $m t$

90 rotamer (Figure 1-Figure supplement 2).

The higher-resolution reconstructions also allowed an improved modelling of ion-binding

92 sites and ordered water molecules in the pore. Two non-protein densities are now resolved that

93 flank the isoleucine constriction in the open state (Figure 1-Figure supplement 2). We assigned

94 these densities as $\mathrm{K}^{+}$ions based on our previous comparison with a structure determined in the

95 presence of $\mathrm{Cs}^{+}$(Oh et al., 2020). These ion-binding sites are positioned $3.7-4.0 \AA$ away from

96 the side chains of Ile46 and Ile271 and are coordinated exclusively by water molecules (Figure

97 1-Figure supplement 2). The binding site on the cytoplasmic site of the constriction is

98 coordinated by four ordered water molecules that are between 2.8 and $3.0 \AA$ away, while the site

99 on the luminal side is coordinated by four water molecules that are between 2.9 and $3.5 \AA$ away.

100 The distance between the two sites is $2.8 \AA$ and thus they are unlikely to be simultaneously

101 occupied by ions. 
For the closed state, we can now model three ion-binding sites and 25 water molecules

103 into non-protein map densities (Figure 1D). In the cytosolic region of the pore, where the

104 channel undergoes minimal conformational changes during gating, the ion-binding sites and

105 water molecules occupy almost identical positions to those resolved in the open state (Figure 1-

106 Figure Supplement 2). In contrast, there is little correspondence between the ion and water

107 configurations at the isoleucine constriction or on the luminal side of the pore due to the

108 conformational changes that occur to the channel during channel gating. The inward movement

109 of Ile271 displaces the ion-binding sites on either side of the isoleucine constriction. Movements

110 of Thr49 and Thr274, which both coordinate water molecules in the open state, result in a

111 movement of the luminal ion-binding site towards the luminal entrance of the pore. Thus, the

112 higher-resolution structures reveal how changes in the protein structure alter the ion conduction

113 pathway to prevent ion permeation in the closed state.

Energetics and mechanism of $K^{+}$permeation

116 To ascertain whether the structure of the putative open state is indeed permeable to $\mathrm{K}^{+}$we

117 turned to all-atom MD simulations of the channel in a model phospholipid bilayer (Figure 2-

118 Figure Supplement 1). Specifically, using the enhanced-sampling methodology known as

119 multiple-walker Metadynamics (Raiteri et al., 2006), we induced the permeation of $\mathrm{K}^{+}$across the

120 channel at $0 \mathrm{mV}$ and symmetric $100 \mathrm{mM} \mathrm{KCl}$ and evaluated the associated potential of mean

121 force (or free-energy profile). Importantly, these calculations used a newly formulated reaction

122 coordinate (Methods) designed to avoid a priori assumptions about the mechanism of

123 permeation, such as the number of ions that reside within the pore at a given time. 
125 ions reach the isoleucine constriction readily from either side of the membrane, through a series

126 of shallow free-energy barriers and transient binding sites, of about $1 \mathrm{kcal} / \mathrm{mol}$ or less (Figure

127 2A). At the isoleucine constriction, however, the free energy increases steeply, peaking at about

$1287 \mathrm{kcal} / \mathrm{mol}$ (Figure $2 \mathbf{A}$ ). $\mathrm{K}^{+}$ions enter and cross the pore individually; unlike for canonical $\mathrm{K}^{+}$

129 channels, we detected no evidence of a multi-ion process (Figure 2-Figure Supplement 2). It is

130 apparent, though, that permeation requires a gradual but drastic depletion of the ion hydration

131 shells. As $\mathrm{K}^{+}$traverses the isoleucine constriction, the first two hydration shells are reduced only

$132 \sim 9$ molecules, down from 31 in the bulk; the first shell averages to about 4 , down from 7

133 (Figures 2B,C and 3A).

Given the largely hydrophobic nature of the TMEM175 pore, it is logical that the free-

135 energy barrier for permeation is significantly higher than those in canonical $\mathrm{K}^{+}$channels. The

136 finding that this barrier is only $7 \mathrm{kcal} / \mathrm{mol}$ is however intriguing, given that the cost of $\mathrm{K}^{+}$

137 dehydration is much larger than that (up to about $80 \mathrm{kcal} / \mathrm{mol}$ for full dehydration). This

138 observation indicates that other factors must facilitate ion permeation in TMEM175. Indeed,

139 further examination of the simulated trajectories using Poisson theory (Methods) reveals that

140 acidic sidechains at both the luminal and cytosolic entrances of the pore generate a strongly

141 favorable electrostatic field that spans the length of the pore (Figure 2D). Some of these residues

142 also transiently coordinate the permeating ions as they enter and exit the pore, along with a few

143 other carbonyl and carboxyl groups in sidechains and backbone (Figure 2C, Figure 2-Figure

144 Supplement 3). However, for a span of about $15 \AA$ centered at the isoleucine constriction, direct

145 protein contacts are minimal. That the barrier for ion conduction in TMEM175 is surmountable 
146 thus reflects not only the degree of hydration retained by the permeating ions, but also the

147 electrostatic field that attracts them to the pore interior.

$148 \quad$ Integration of the potential-of-mean-force profile obtained at $0 \mathrm{mV}$ and $100 \mathrm{mM} \mathrm{KCl}$

149 translates into a single-channel conductance of approximately $0.2 \mathrm{pS}$ (Methods). At $500 \mathrm{mV}$, and

150 assuming the structure of the channel is completely unresponsive to voltage, this conductance

151 would in turn produce a $0.1 \mathrm{pA}$ current, or a rate of $\mathrm{K}^{+}$permeation of about 0.6 ions/ $\mu$ s. To

152 assess these estimates, we calculated an independent $1-\mu$ s long MD simulation using

153 conventional sampling but under $500 \mathrm{mV}$ (lumen positive) and $400 \mathrm{mM} \mathrm{KCl}$. This trajectory

154 revealed two $\mathrm{K}^{+}$permeation events, confirming that the open state is functionally conductive and

155 validating the single-ion mechanism inferred from the free-energy simulations (Figure 3C,D).

$156 \mathrm{~K}^{+}$ions are seen to dwell at the shallow binding site adjacent to the constriction, on the luminal

157 side, before returning to the bulk or rapidly traversing the free-energy barrier, along with 4 water

158 molecules in the first hydration shell (Figure 3C).

Differentials in ion dehydration relative to bulk explain selectivity for $\mathrm{K}^{+}$over $\mathrm{Na}^{+}$

Like canonical $\mathrm{K}^{+}$channels, TMEM175 channels are more permeable to $\mathrm{K}^{+}$than $\mathrm{Na}^{+}$.

162 However, in the absence of the selectivity filter seen in canonical $\mathrm{K}^{+}$channels, it has been

163 unclear how to explain their selectivity in structural terms. To begin to bridge this gap, we again

164 turned to MD simulations. Specifically, we repeated the enhanced-sampling simulations carried

165 out to examine $\mathrm{K}^{+}$permeation after substituting the $\mathrm{KCl}$ solution with $\mathrm{NaCl}$ and examined the

166 resulting data analogously (Figures 2 and 3). The potential-of-mean-force profile for $\mathrm{Na}^{+}$is

167 similar to that obtained for $\mathrm{K}^{+}$in its overall features, including the rate-limiting barrier at the

168 isoleucine constriction (Figure 2a). The largest difference between the profiles is the magnitude 
169 of the barrier at the isoleucine constriction, which is greater for $\mathrm{Na}^{+}$than for $\mathrm{K}^{+}$, and thus

170 consistent with TMEM175 being a $\mathrm{K}^{+}$selective channel. The barrier peaks at about $8 \mathrm{kcal} / \mathrm{mol}$

171 relative to bulk for $\mathrm{Na}^{+}$compared to about $7 \mathrm{kcal} / \mathrm{mol}$ for $\mathrm{K}^{+}$. As observed for $\mathrm{K}^{+}, \mathrm{Na}^{+}$

172 permeation proceeds via a single-ion mechanism (Figure 2-Figure Supplement 2, Figure 3B).

173 Permeation is again opposed by the drastic dehydration of the ion (Figures 2B,), and favored by

174 its interaction with the electrostatic field created by the protein within the pore (Figure 2D), as

175 well as by transient interactions with multiple residues on both cytoplasmic and luminal sides

176 (Figure 2C). These stabilizing factors are not identical but appear comparable to those observed

177 for $\mathrm{K}^{+}$. By contrast, the degree of dehydration required for permeation clearly differs. We

178 observe that the depletion in the both the first and second solvation shells, relative to bulk

179 numbers, is significantly smaller for $\mathrm{Na}^{+}$than for $\mathrm{K}^{+}$. As will be further discussed below, this

180 relative difference in dehydration energetics likely explains why TMEM175 is only $~ 30$-fold

181 more permeable to $\mathrm{K}^{+}$, even though the bulk water selectivity for $\mathrm{Na}^{+}$is over a trillion-fold. In

182 other words, like for $\mathrm{K}^{+}$channels that feature a canonical selectivity filter, it can be said that the

183 narrow pore of TMEM175 favors $\mathrm{Na}^{+}$over $\mathrm{K}^{+}$, but not as much as bulk water. The channel is

184 thus more permeable to $\mathrm{K}^{+}$than $\mathrm{Na}^{+}$.

186 Role of the isoleucine constriction in ion selectivity and permeation

187 The isoleucine constriction constitutes the largest free-energy barrier to the permeation of

$188 \mathrm{~K}^{+}$and $\mathrm{Na}^{+}$in the pore of TMEM175 (Figure 2). This narrow constriction forces the ions to shed

189 much of their hydration shells in order to traverse the pore. It was previously demonstrated that

190 mutating Ile46 and Ile271 to asparagine diminishes the selectivity of TMEM175, indicating that

191 the hydrophobicity of the isoleucine constriction is crucial for selectivity (Lee et al., 2017). To 
192 determine if the dimensions of the constriction are also important, we used MD simulations to

193 calculate the change in free energy that results when a $\mathrm{K}^{+}$ion at the isoleucine constriction is

194 exchanged with a $\mathrm{Na}^{+}$ion, both for wild-type TMEM175 and for a I46V mutant with an

195 expanded isoleucine constriction (Methods). In both cases the exchange results in a free-energy

196 gain (Figure 4A), consistent with the notion that $\mathrm{Na}^{+}$is the favored species at the constriction;

197 this gain is however smaller than that observed for bulk water, which as discussed is the

198 expected result for a $\mathrm{K}^{+}$selective channel (Figure 4A). Specifically, the differential relative to

199 bulk is $1.2 \mathrm{kcal} / \mathrm{mol}$ in favor of $\mathrm{K}^{+}$, similar to the $\sim 1 \mathrm{kcal} / \mathrm{mol}$ difference in the peak values of the

200 free-energy curves discussed in the previous section (Figure $\mathbf{2 A}$ ). $\mathrm{Na}^{+}$is however favored to a

201 larger extent in the I46V mutant, whose isoleucine constriction has a similar hydrophobicity but

202 is expanded by the loss of a methyl group from the side chain of Ile46 in each protomer (Figure

203 4A); specifically, the calculations indicate this mutant is about 2-fold less $\mathrm{K}^{+}$selective than the

204 wild-type channel (Figure 4A). These results again correlate with the degree of dehydration of

205 each ion, relative to bulk values (Figure 4B).

206 Using whole-cell electrical recordings, we experimentally assessed the effects of

207 expanding the size of the isoleucine constriction on selectivity by measuring the selectivity of

$208 \mathrm{Cs}^{+}$over $\mathrm{Na}^{+}$for comparison with the computational analyses. We choose $\mathrm{Cs}^{+}$rather than $\mathrm{K}^{+}$

209 because $\mathrm{Cs}^{+}$blocks most canonical $\mathrm{K}^{+}$channels, thereby reducing endogenous currents.

210 Nevertheless, whole-cell currents recorded from cells expressing the I46A, I46V, I271A and

211 I271V mutants in a bi-ionic $\mathrm{Cs}^{+} / \mathrm{Na}^{+}$condition were much smaller than those recorded from

212 wild-type TMEM175 and in some cases similar to currents recorded from nontransfected cells,

213 which hampered accurate determination of ion permeability ratios via measuring reversal

214 potentials $\left(\mathrm{E}_{\mathrm{rev}}\right)$ (Figure 5 and Figure 5-Figure supplement 3). Thus, isolation of the 
215 TMEM175-specific currents from the endogenous HEK293T and background leak was

216 necessary to evaluate the selectivity of the TMEM175 mutants. To this end, we first performed a

217 background subtraction with the TMEM175 inhibitor 4-aminopyridine (4-AP) (Cang et al.,

218 2015). However, while 4-AP can effectively inhibit wild-type TMEM175, it was ineffective

219 against the mutants and thus not suitable for isolating the TMEM175-specific currents (Figure 5-

220 Figure supplements 3 and 4). We therefore repeated the background subtraction experiments

221 with a novel TMEM175 inhibitor that we have developed, which we call AP-6 (Figure 5 and

222 Figure 5-Figure supplements 1-3). Following AP-6 background subtraction, I46V and I271V

223 displayed $\mathrm{Cs}^{+}$-selective currents with $\mathrm{E}_{\text {rev }}$ of $-13 \mathrm{mV}$ and $-18 \mathrm{mV}$, corresponding to $\mathrm{Cs}^{+}$over $\mathrm{Na}^{+}$

224 permeability ratios $\left(\mathrm{P}_{\mathrm{Cs} / \mathrm{Na}}\right)$ of 1.7 and 2.0, respectively (Figure 5). Using the same background

225 subtraction approach, we estimated the $\mathrm{P}_{\mathrm{Cs} / \mathrm{Na}}$ of wild-type TMEM175 to be 20 ( $\mathrm{E}_{\mathrm{rev}}$ of $\left.-76 \mathrm{mV}\right)$.

226 This 10 -fold reduction in $\mathrm{P}_{\mathrm{Cs} / \mathrm{Na}}$ ion permeation ratio is in line with the computational analyses of

227 free energy difference in resulting from $\mathrm{K}^{+}$to $\mathrm{Na}^{+}$ion exchange at the isoleucine constriction of

228 I46V and WT. We were not able to detect any AP-6 sensitive currents from the I46A or I271A

229 mutants either due their insensitivity towards AP-6 or inactivity of the mutant channels. These

230 data demonstrate that increasing the size of the isoleucine constriction leads to a clear reduction

231 in ion selectivity. Thus, the isoleucine constriction, which is universally conserved among

232 eukaryotic TMEM175 channels, serves as a hydrophobic selectivity filter that is exquisitely

233 sensitive to very subtle changes in its structure.

Discussion

Permeation through selective ion channels arises from the interplay between channel-ion

237 interactions, solvent-ion interactions, and in some cases, ion-ion interactions. The energetic cost

238 associated with fully or partially dehydrating an ion such as $\mathrm{K}^{+}$is so large that permeation would 
only very rarely occur without favorable interactions with the channel as the ion traverses the conduction pathway. In canonical $\mathrm{K}^{+}$channels backbone and side-chain oxygen atoms provide a

241 series of direct coordinating interactions that closely resemble those experienced by the ion in 242 bulk water. In addition, these channels are often capable of attracting multiple ions to the pore 243 concurrently. Thus, the cost of dehydration is almost entirely offset by channel-ion and ion-ion 244 interactions, and the resulting free-energy barriers for permeation are small. In contrast, such 245 interactions are lacking for much of the TMEM175 pore, and entirely absent near the isoleucine 246 constriction. $\mathrm{K}^{+}$ions crossing the pore must nevertheless shed much of their hydration shells; 247 only $\sim 9$ of the 31 water molecules in its first two shells remain at the constriction. In addition, 248 ions permeate TMEM175 one at a time, without the aid of knock-on interactions. Permeation is 249 however strongly favored by a delocalized electrostatic field across the pore, which offsets much 250 of the cost of ion dehydration, resulting in a free-energy barrier of $\sim 7 \mathrm{kcal} / \mathrm{mol}$ (Figure 2). While 251 this is a high barrier, it is not insurmountable. Indeed, considering that MD simulations using 252 conventional forcefields tend to underestimate ion conduction rates, in some cases up to 10-fold 253 (Allen et al., 2006; Mironenko et al., 2021; Zhu and Hummer, 2012), we estimate the unitary 254 conductance of human TMEM175 is $0.1-0.5 \mathrm{pS}$, which is well within the broad range of 255 experimentally measured permeation rates for other selective cation channels such as Slo $\mathrm{K}^{+}$and 256 CRAC channels (Prakriya et al., 2006; Tao et al., 2017). Thus, despite the hydrophobicity of the 257 isoleucine constriction, the unique architecture and amino-acid make-up of the TMEM175 pore 258 facilitates ion permeation.

260 dependent on the ion type, and so these opposing forces also govern ion selectivity in channels.

261 For example, when rationalizing differences in permeability between $\mathrm{K}^{+}$and $\mathrm{Na}^{+}$, it is useful to 
262 keep in mind that dehydration of $\mathrm{Na}^{+}$is much more costly than that of $\mathrm{K}^{+}$; the penalty for full

263 dehydration is $\sim 18 \mathrm{kcal} / \mathrm{mol}$ greater, rendering it a trillion-fold less probable. However, the

264 observed selectivity of biological $\mathrm{K}^{+}$channels against $\mathrm{Na}^{+}$is only 1000 -fold or less, implying

$265 \mathrm{Na}^{+}$establishes much more favorable interactions with the selectivity filters of this kind of $\mathrm{K}^{+}$

266 channels. In other words, what ultimately determines the specificity of a channel is the sum of

267 the relative cost of dehydration of the competing ions and the differential in their free energy of

268 interaction with the channel. Remarkably, for TMEM175 these favorable protein-ion interactions

269 are imparted by residues near the luminal and cytoplasmic entrances to the pore, quite far from

270 the isoleucine constriction. Thus, we conclude that the ion selectivity of TMEM175 primarily

271 results from a differential in the degree of dehydration required for permeation, relative to bulk

272 water.

Together, our analyses provide a structural and energetic basis for the unique

274 mechanisms of ion permeation and selectivity in TMEM175 channels. By combining a narrow

275 hydrophobic sieve with a favorable electrostatic field, TMEM175 selectively permeates $\mathrm{K}^{+}$ions

276 across the lysosomal membrane, albeit at a rate that is 100-1000 times lower than most canonical

$277 \mathrm{~K}^{+}$channels. As the large conductance Slo1 $\mathrm{K}^{+}$channel has also been observed in lysosomes, it

278 will be important to uncover the specific roles for TMEM175's distinct conduction and

279 selectivity properties in the lysosome and why mutations of TMEM175 can both increase and

280 decrease the likelihood of developing PD (Wang et al., 2017; Zhong et al., 2016). 


\section{Methods}

282

283

284

285

286

287

288

289

290

291

292

293

294

295

296

297

298

299

300

301

302

303

304

305

306

307

308

309

310

\section{Analysis of Electron microscopic images}

7,907 images of TMEM175 in $\mathrm{KCl}$ previously acquired in two data sets as 40-frame super-resolution movies (0.544 $\AA$ /pixel) using a Gatan K2 (Oh et al., 2020) were were gain corrected, Fourier cropped by two and aligned using whole-frame and local motion correction algorithms by Motioncor2 (Zheng et al., 2017) (1.088 §/pixel). Whole-frame CTF parameters were determined using CTFfind 4.1.14 (Rohou and Grigorieff, 2015). Particles were automatically selected in Relion 3.0 using templates previously generated from 2D classification, resulting in 4,153,614 particles (Zivanov et al., 2018). False-positive selections and contaminants were excluded from the data using multiple rounds of heterogeneous classification in cryoSPARC v3.2 using the open and closed states, as well as several decoy classes generated from noise particles via ab initio reconstruction in cryoSPARC v3.2, resulting in a stack of 636,148 particles (Punjani et al., 2017). After Bayesian polishing in Relion and local CTF estimation and higher order aberration correction in cryoSPARC v3.2, a consensus reconstruction was determined at resolution of $2.8 \AA$ (Zivanov et al., 2019). A second round of Bayesian polishing in Relion 3 using a pixel size of $0.85 \AA$ and box size of 384 yielded an improved consensus reconstruction at $2.5 \AA$. Iterative supervised heterogenous classification using open and closed maps low pass filtered to $6 \AA$ as references resulted in 261,536 particles in the open state and 163,651 particles in the closed state. A $2.45 \AA$ reconstruction of the open state particles and a $2.61 \AA$ reconstruction of the closed state particles were obtained using nonuniform refinement in cryoSPARC v3.2 employing global and per-particle CTF correction. The reconstructions were subjected to density modification using the two unfiltered half-maps with a soft mask in Phenix (Terwilliger et al., 2019).

\section{Model building and coordinate refinement}

The structures of open (PDB:6WC9) and closed (PDB:6WCA) human TMEM175 were docked into the density maps in COOT and manually adjusted to fit the density (Emsley et al., 2010). Densities corresponding to TM5 and TM6 (residues 174-251) were too poorly ordered and omitted from the model. The final models are composed of residues 30-173, and 254-476. Atomic coordinates were refined against the density modified map using 
311 phenix.real_space_refinement with geometric and Ramachandran restraints maintained

312 throughout (Adams et al., 2010).

314 Simulation systems and general specifications

315 All simulations were calculated with NAMD 2.12 using the CHARMM36 force field for 316 protein and lipids (Best et al., 2012; Klauda et al., 2010; Pastor and MacKerell, 2011; Phillips et 317 al., 2005). The simulations were carried out at constant temperature (298 K) and semi-isotropic 318 pressure (1 atm), using periodic boundary conditions and an integration time step of 2 fs. Long319 range electrostatic interactions were calculated using PME, with a real-space cut-off of $12 \AA$. Van 320 der Waals interactions were computed with a Lennard-Jones potential, cut-off at $12 \AA$ with a 321 smooth switching function taking effect at $10 \AA$.

322 The simulations were based on the high-resolution cryo-EM structure of the human 323 TMEM175 channel (PDB 6WC9). The specific construct studied includes residues 30-165 and 324 residues $254-476 . \mathrm{K}^{+}$ions and water molecules originally included in the cryo-EM structure were removed. All ionizable sidechains were set in their default protonation state at $\mathrm{pH} 7$, except for $326 \mathrm{H} 57$, which was protonated on account of its proximity to D279, E282 and D283. The protein 327 construct was embedded in a pre-equilibrated hydrated palmitoyl-oleoyl-phosphatidyl-choline 328 (POPC) lipid bilayer using GRIFFIN, and enclosed in a periodic orthorhombic box of $\sim 100 \times 100$ $329 \times 111 \AA$ in size (Staritzbichler et al., 2011). The two resulting simulation systems contain 222 330 POPC lipids, 24342 water molecules, $49 \mathrm{Cl}^{-}$ions and either $43 \mathrm{~K}^{+}$or $43 \mathrm{Na}^{+}$ions; that is, a salt 331 concentration of $100 \mathrm{mM}$ plus counterions to neutralize the protein net charge. The simulation 332 systems were equilibrated following a staged protocol comprising a series of restrained 333 simulations. The protocol consists of both positional and conformational restraints, gradually 334 weakened over $100 \mathrm{~ns}$. A third simulation system was prepared with $400 \mathrm{mM} \mathrm{KCl}$, by adding 132 $335 \mathrm{~K}^{+}$and $132 \mathrm{Cl}^{-}$ions to the $100 \mathrm{mM} \mathrm{KCl}$ system (replacing water), followed by a 100-ns 336 equilibration.

Simulation of $\mathrm{K}^{+}$permeation under voltage

To simulate the flow of $\mathrm{K}^{+}$across TMEM175 from the luminal to the cytoplasmic side, a conventional MD trajectory of $1 \mu$ s was calculated for the $400 \mathrm{mM} \mathrm{KCl}$ condition under a constant 341 electric field perpendicular to the membrane plane, of magnitude $E_{\mathrm{z}}=-0.1045 \mathrm{kcal} /(\mathrm{mol} \AA \mathrm{e})$. 
342 Given the average length of the box in this direction $\left(L_{\mathrm{z}}=110.5 \AA\right)$, the corresponding transmembrane potential is $-500 \mathrm{mV}\left(\Phi=E_{\mathrm{z}} \times L_{\mathrm{z}} \times 0.0434 \mathrm{~V} / \AA\right)$ (Roux, 2008). $\mathrm{mM} \mathrm{NaCl}$, we used the multiple-walker Metadynamics method (Raiteri et al., 2006). In Metadynamics, a biasing potential is introduced in a MD simulation to facilitate the exploration of configurational space, as defined by one or more reaction coordinates, also known as collective variables (CVs). This biasing potential consists of a series of Gaussian functions that expands as the simulation progresses, to foster the trajectory to visit high free-energy configurations. In the multiple-walker approach, several MD simulations are carried out in parallel, each sampling a different trajectory, but with a shared biasing potential that is also collectively constructed. To study $\mathrm{K}^{+}$permeation, we used 8 walkers, each producing a 600 -ns trajectory each. For $\mathrm{Na}^{+}$, we also used 8 walkers, 700 ns each.

The type of collective variable used in these simulations was newly formulated to circumvent a priori assumptions regarding the mechanism of ion permeation. This variable was implemented in a modified version of PLUMED 1.3 (Bonomi et al., 2009). Specifically, the variable is a quantitative descriptor of the proximity between the ions in the simulation system and a virtual center within the pore. More precisely, the variable is:

$$
\zeta_{\min }=\frac{\beta}{\log \sum_{k} \exp \left\{\beta /\left(\left|\Delta Z_{k}\right|+C\right)\right\}}-C
$$

361 where the $k$ index identifies each of the $\mathrm{K}^{+}$or $\mathrm{Na}^{+}$ions in the system and $\Delta Z_{k}$ is the cartesian Z362 component of the distance between ion $k$ and a center-of-mass defined by a group of protein atoms. $363 \mathrm{C}$ is a positive constant (set to $2 \AA$ ) that avoids numerical instabilities when $\Delta Z_{k} \sim 0$ and $\beta$ is a 364 smoothing parameter (set to $100 \AA$ ). To successfully induce ion permeation events, we used two 365 variables of the kind specified by Eq. 1, namely $\zeta_{\min }^{\mathrm{A}}$ and $\zeta_{\mathrm{min}}^{\mathrm{B}}$, each defined in reference to a 366 center of atoms. Center $\mathrm{A}$ is defined by the $\mathrm{C} \alpha$ atoms of residues 44-47 and 268-271, while center $367 \mathrm{~B}$ is defined by the $\mathrm{C} \alpha$ atoms of residues 45-48 and 269-272. Centers $\mathrm{A}$ and $\mathrm{B}$ are therefore along 368 the pore axis, separated by $1 \AA$ and flanking the isoleucine constriction. The Gaussian functions 369 used to gradually construct the biasing potentials applied to $\zeta_{\min }^{\mathrm{A}}$ and $\zeta_{\min }^{\mathrm{B}}$ had a width $0.25 \AA$ and 
370

371

372

373

374

375

376

377

378

379

380

381

382

383

384

385

386

were added in 4-ps intervals. To reduce systematic errors near the boundary $\zeta_{\min }=0$, reflected and inverted Gaussians were added beyond this boundary for both variables as previously described (Crespo et al., 2010). In the simulations of $\mathrm{K}^{+}$permeation, the Gaussians height was gradually raised from $0.0035 \mathrm{kcal} / \mathrm{mol}$ to $0.007 \mathrm{kcal} / \mathrm{mol}$ in the first $30 \mathrm{~ns}$ of simulation, and gradually diminished back to $0.0035 \mathrm{kcal} / \mathrm{mol}$ after $100 \mathrm{~ns}$. For $\mathrm{Na}^{+}$, the Gaussians height was gradually raised from $0.0035 \mathrm{kcal} / \mathrm{mol}$ to $0.007 \mathrm{kcal} / \mathrm{mol}$ in the first $50 \mathrm{~ns}$ of simulation and diminished back to $0.0035 \mathrm{kcal} / \mathrm{mol}$ after $250 \mathrm{~ns}$.

\section{Derivation of free energies and other quantitative descriptors from biased-sampling trajectories}

A post-hoc reweighting procedure was used to derive unbiased averages and histograms from the MD trajectories enhanced by the Metadynamics biasing potential (Marinelli et al., 2009). In this approach, a time-average of the Metadynamics biasing potential is calculated after it becomes approximately stationary across the range of $\left(\zeta_{\min }^{\mathrm{A}}, \zeta_{\mathrm{min}}^{\mathrm{B}}\right)$ values of interest. (Here, the last $400 \mathrm{~ns}$ of simulation for $\mathrm{K}^{+}$and the last $600 \mathrm{~ns}$ of simulation for $\mathrm{Na}^{+}$). This effective potential, $\bar{V}\left(\zeta_{\min }^{\mathrm{A}}, \zeta_{\mathrm{min}}^{\mathrm{B}}\right)$, is then used to adjust the statistical weight of each of the simulation snapshots considered in the analysis. Specifically, the statistical weight of a given snapshot $\boldsymbol{X}_{i}$ is:

$$
w\left(\boldsymbol{X}_{i}\right)=\frac{\exp \left\{\bar{V}\left(\zeta_{\min }^{\mathrm{A}}\left(\boldsymbol{X}_{i}\right), \zeta_{\min }^{\mathrm{B}}\left(\boldsymbol{X}_{i}\right)\right) / k_{\mathrm{B}} T\right\}}{\sum_{j} \exp \left\{\bar{V}\left(\zeta_{\min }^{\mathrm{A}}\left(\boldsymbol{X}_{j}\right), \zeta_{\min }^{\mathrm{B}}\left(\boldsymbol{X}_{j}\right)\right) / k_{\mathrm{B}} T\right\}}
$$

where $k_{\mathrm{B}}$ is the Boltzmann constant and $T$ is the temperature. That is, snapshots $\boldsymbol{X}_{i}$ that fall in easily accessible regions of $\left(\zeta_{\mathrm{min}}^{\mathrm{A}}, \zeta_{\mathrm{min}}^{\mathrm{B}}\right)$ where the accumulated Metadynamics bias is large are given a greater statistical weight, while those in unfavorable regions where the accumulated bias is less are also assigned a smaller weight.

Following this approach, it is straightforward to derive unbiased estimates of the average ion occupancy along the length of the channel pore, and thus of the corresponding free energy landscape. Specifically, let us define this length by an axis connecting the abovementioned centers $\mathrm{A}$ and $\mathrm{B}$ (which fluctuates with the channel but is approximately perpendicular to the membrane plane). For each ion $k$ in the simulation system, let us also define $z_{k}\left(\boldsymbol{X}_{i}\right)$ and $R_{k}\left(\boldsymbol{X}_{i}\right)$ as the projection of the ion coordinates along the pore axis and the distance to that axis, respectively, for a given snapshot $\boldsymbol{X}_{i}$. If we divide the pore axis into a series of intervals or bins, the ion occupancy of a given bin $\alpha$ centered in $z_{\alpha}$ is: 


$$
\rho\left(z_{\alpha}\right)=\sum_{i} \sum_{k \in\left(\alpha, R_{0}\right)} w\left(\boldsymbol{X}_{i}\right)
$$

where the $j$ index denotes all possible coordinating oxygens, and $r_{j k}\left(\boldsymbol{X}_{i}\right)$ denotes their distance to

where the $i$ index again denotes each trajectory snapshot included in the analysis, and the sum over $k$ is restricted to ions whose $z_{k}\left(\boldsymbol{X}_{i}\right)$ coordinate falls into bin $\alpha$ and that are also proximal to the pore axis $\left(R_{k}\left(\boldsymbol{X}_{i}\right) \leq R_{0}=10 \AA\right)$. It follows that the potential of mean force (or free energy profile) associated with this occupancy distribution along $z_{\alpha}$ will be:

$$
F\left(z_{\alpha}\right)=-k_{\mathrm{B}} T \log \rho\left(z_{\alpha}\right)=-k_{\mathrm{B}} T \log \sum_{i} \sum_{k \in \alpha} w\left(\boldsymbol{X}_{i}\right)+C^{\prime}
$$

where $C^{\prime}$ is a constant arbitrarily selected so that $F \sim 0$ in the bulk, where the profile is nearly flat.

We followed same reweighting approach to derive unbiased estimates for other descriptors, namely average ion-coordination numbers and average ion-protein electrostatic interaction energies (definitions provided below). Specifically, the mean value of either of these observables at a given position along the pore axis is:

$$
\bar{O}\left(z_{\alpha}\right)=\frac{1}{\rho\left(z_{\alpha}\right)} \sum_{i} \sum_{k \in\left(\alpha, R_{0}\right)} O_{k}\left(\boldsymbol{X}_{i}\right) w\left(\boldsymbol{X}_{i}\right)
$$

where $i$ again denotes a trajectory snapshot, $O_{k}\left(\boldsymbol{X}_{i}\right)$ is the descriptor of interest of ion $k$, in that particular snapshot, and the sum over $k$ is again restricted to ions within the pore and within bin $\alpha$.

\section{Calculation of ion coordination numbers}

To quantify the coordination of $\mathrm{K}^{+}$or $\mathrm{Na}^{+}$by either water or protein oxygen atoms as a function of the ion position along the pore axis, we evaluated the following step function of the ion-oxygen distances for each simulation snapshot $\boldsymbol{X}_{i}$ and each ion $k$ in the system:

$$
S_{k}\left(\boldsymbol{X}_{i}\right)=\frac{\sum_{j} 1-\left(r_{j k}\left(\boldsymbol{X}_{i}\right) / r_{0}\right)^{100}}{\sum_{j} 1-\left(r_{j k}\left(\boldsymbol{X}_{i}\right) / r_{0}\right)^{200}}
$$

ion $k$ in snapshot $\boldsymbol{X}_{i}$. For the evaluation of the number of water molecules in the first hydration shell, $r_{0}$ was set to $3.5 \AA$ for $\mathrm{K}^{+}$and to $3.2 \AA$ for $\mathrm{Na}^{+}$, which in each case corresponds to the position of the first minimum of the ion-oxygen radial distribution functions (RDF) in bulk water (data not shown). The same values of $r_{0}$ were used for calculating the coordination with protein oxygens. Following the same criteria, the number of water molecules in the first and second shells was calculated by setting $r_{0}$ to $6.0 \AA$ and $5.7 \AA$, respectively, as these values reflect the position of the 
421 second minimum of the RDF in each case. The two exponents of the step function in Eq. 6 were set to reproduce the integral of the RDF function in bulk water within $r_{0}$.

\section{Calculation of ion-protein electrostatic interaction energies}

To estimate the electrostatic interaction energy between each $\mathrm{K}^{+}$or $\mathrm{Na}^{+}$ion and the channel, we used a continuum electrostatic model based on the linearized form of Poisson's equation. That is, for each ion $k$ and snapshot $\boldsymbol{X}_{i}$, we evaluated the electrostatic potential $\Phi_{k}$ generated at the position of the ion by the atomic charges in the protein structure $\left(q_{p}\right)$, in the context of a heterogenous dielectric-constant distribution defined by the protein $\left(\varepsilon_{p}=2\right.$ or 1$)$, an implicit membrane surrounding the protein $\left(\varepsilon_{m}=2\right)$, the bulk water solvent $\left(\varepsilon_{s}=80\right)$, the water solvent inside the pore $\left(\varepsilon_{S^{\prime}}=40\right)$ and the ion in question $\left(\varepsilon_{k}=2\right.$ or 1$)$. (Note that both charge and dielectricconstant distributions are a function of $\boldsymbol{X}_{i}$, as is the position of the ion.) The interaction between this electrostatic potential and the ion is given by:

$$
E_{k}\left(\boldsymbol{X}_{i}\right)=q_{k} \Phi_{k}\left(q_{p}, \varepsilon_{p}, \varepsilon_{m}, \varepsilon_{s}, \varepsilon_{s^{\prime}}, \varepsilon_{k} ; \boldsymbol{X}_{i}\right)
$$

These calculations were carried out with the PBEQ numerical solver implemented in CHARMM c44b1 (Brooks et al., 2009). The set of atomic charges employed are those in the CHARMM36 force field; the set of atomic radii used to define the dielectric boundaries derives from an existing optimization for continuum-electrostatic calculations based on the CHARMM27 force field (Best et al., 2012; Nina et al., 1997). Electrostatic potential, charge and dielectric-constant distributions were discretized on a lattice of $150 \times 150 \times 150 \AA$, with lattice-point spacing of $1 \AA$. The thickness of the implicit membrane was $28.8 \AA$. The low-dielectric span of pore water was defined by a

441 cylinder of radius $18 \AA$ and height $28.8 \AA$. The protein surface was defined using the REEN 442 method, using a probe radius of $1 \AA$.

\section{Calculation of ion conductance}

To obtain an approximate estimate of the $\mathrm{K}^{+}$conductance of TMEM175 channel, we used 446 the theoretical formulation proposed by Zhu and Hummer (Zhu and Hummer, 2012), wherein the conductance $\gamma$ is inferred from the free-energy $(F(z))$ and diffusion $(D(z))$ profiles as a function of the ion position along the pore axis: 


$$
\gamma=\frac{q^{2} C S}{k_{\mathrm{B}} T}\left[\int_{z_{1}}^{z_{2}} \exp \left\{\frac{F(z)}{k_{\mathrm{B}} T}\right\} \frac{1}{D(z)} d z\right]^{-1}
$$

where $q(=1)$ is the charge of the permeant ion, $C$ is the bulk ion concentration $(100 \mathrm{mM})$, and $S$ is the cross-section area $\left(314 \AA^{2}\right)$ of the cylindrical region considered to evaluate Eqs. 3-5. As a first approximation, we assumed the diffusion profile be flat, and used $D(z) \sim 210^{-9} \mathrm{~m}^{2} / \mathrm{s}$ following a previous simulation study (Zhou et al., 2017). Note that $\gamma$ depends linearly with $D(z)$, while the dependence on $F(z)$ is exponential; thus, while it is very plausible that the diffusion constant will vary by at least a factor of 2 as the ion traverses the pore, the order of magnitude of the conductance is largely set by the features of the free-energy profile.

\section{Calculation of free energies of selectivity}

To evaluate free-energy differences between $\mathrm{K}^{+}$and $\mathrm{Na}^{+}$states, whether in bulk water or at the isoleucine constriction within the TMEM175 pore, we used the Free-Energy Perturbation (FEP) method as implemented in NAMD 2.12. To ascertain the magnitude of sampling errors, all transformations were carried out in the forward $\left(\mathrm{K}^{+}\right.$to $\left.\mathrm{Na}^{+}\right)$and backward $\left(\mathrm{K}^{+}\right.$to $\left.\mathrm{Na}^{+}\right)$directions. The transformations were carried out in 26 steps; each step included a 50-ps equilibration, excluded from analysis, followed by 500 ps of averaging time. For bulk water, the coupling parameter lambda was varied increments of 0.04 in the [0, 0.8] interval and in increments of 0.02 in the $[0.8,1]$ interval. For the protein calculations, lambda was changed in increments of 0.04 in the $[0,0.96]$ interval and in increments of 0.02 in the $[0.96,1]$ interval. To ensure that the $\mathrm{K}^{+}$and $\mathrm{Na}^{+}$ions involved in the transformation are in the same position, the distance between the two particles is restrained to zero using a harmonic potential of force constant $2425.58 \mathrm{kcal} /\left(\mathrm{mol} \AA^{2}\right)$. In the protein calculations, the ion(s) are confined to remain within the isoleucine constriction using a flat-bottom distance restraint, defined relative to the center-of-mass of the backbone atoms of two groups of residues, namely 44-45, 269-270 (first group) and 47-49, 272-274 (second group). These two groups also define an axis; the restraint acts on the distance between the ion and the center-of-mass, as projected on that axis. The confining potential is flat-bottomed, permitting fluctuations of $\pm 0.1 \AA$ in the distance, but further deviations are suppressed with a harmonic function of force constant $100 \mathrm{kcal} /\left(\mathrm{mol} \AA^{2}\right)$. 
Electrophysiological analysis

Electrophysiological recordings of TMEM175 constructs were performed in HEK293T cells (ATCC CRL-3216). HEK293T cells cultured in DMEM supplemented with 10\% FBS. To

480 transfer cells in single dishes, cells were detached by trypsin treatment. The detached cells were transferred to poly-Lys-treated $35 \mathrm{~mm}$ single dishes (FluoroDish, World Precision Instruments) and incubated overnight at $37^{\circ} \mathrm{C}$ in fresh media. Cells in a single dish were transfected with 1.25

$483 \mu \mathrm{g}$ of c-term EGFP tagged hTMEM175 plasmid using $3.75 \mu \mathrm{g}$ of PEI $25 \mathrm{k}$ (Polysciences, Inc).

484 Electrophysiological recordings were performed 48-72 hr after transfection. Prior to recording, media was replaced with a bath solution containing $145 \mathrm{mM}$ Na-methanesulfonate (MS), $5 \mathrm{mM}$ $\mathrm{NaCl}, 1 \mathrm{mM} \mathrm{MgCl} 2,1 \mathrm{mM} \mathrm{CaCl}$, $10 \mathrm{mM}$ HEPES/Tris pH 7.4. $10 \mathrm{~cm}$ long borosilicate glasses were pulled and fire polished (Sutter instrument). The resistance of glass pipette was $3 \sim 10 \mathrm{M} \Omega$ were filled with a pipette solution containing $150 \mathrm{mM} \mathrm{Cs-MS,} 5 \mathrm{mM} \mathrm{MgCl}$, $10 \mathrm{mM}$ EGTA, 10 $\mathrm{mM}$ HEPES/Tris $\mathrm{pH} 7.4$, and $\mathrm{G} \Omega$ seals were formed after gentle suction. The recordings were performed in whole cell patch clamp configuration using the following protocol: from a holding potential of $0 \mathrm{mV}$, the voltage was stepped to voltages between -100 and $+100 \mathrm{mV}$, in $20 \mathrm{mV}$

492 increments. To measure currents reduced by AP-6, bath solutions were perfused. DMSO

493 concentration was maintained as $0.33 \%$ during the entire experiment. The currents were

494 recorded using Axon Digidata 1550B digitizer and Clampex 10.6 (Molecular Devices, LLC) and 495 analyzed using AxoGraph X 1.7.6 (AxoGraph Scientific). Each experiment was performed in a 496 unique cell.

\section{General Chemistry}

499 Chemical reagents and materials were purchased from Sigma-Aldrich, Thermo-Fischer, 500 and TCI, and used without further purifications. DCM (dichloromethane), $\mathrm{MeOH}$, and $n$-hexane 501 for column chromatography and recrystallization were used for HPLC grade without additional 502 purifications. Thin layer chromatography (TLC) analysis was performed for reaction monitoring 503 on the pre-coated silica gel 60 F254 glass plates. Both starting materials and the desired product 504 were checked by UV light (254 nm). Flash column chromatography was carried out on silica gel 505 (400-630 mesh) to separate the target molecule. 
AP-6 was prepared through Suzuki-Miyaura cross-coupling reaction with palladium catalysis. In a round-bottom flask with a magnetic bar, a mixture of 2-bromopyridin-4-amine (5 mmol, $865 \mathrm{mg}$ ), 1,3-phenylenediboronic acid (0.75 equiv., $622 \mathrm{mg}), \mathrm{K}_{2} \mathrm{CO}_{3}$ (2 equiv., $1.38 \mathrm{~g}$ ),

511 and $\mathrm{Pd}(\mathrm{OAc})_{2}(7 \mathrm{~mol} \%, 78.6 \mathrm{mg})$ were dissolved in $\mathrm{H}_{2} \mathrm{O}:$ EtOH solution mixture $(8 \mathrm{~mL}: 32 \mathrm{~mL})$,

512 and the solution was stirred at $100{ }^{\circ} \mathrm{C}$ for $24 \mathrm{~h}$ under air. After completion (monitored by TLC),

513 the reaction mixture was filtered through a Celite (after cooling to room temperature), and then

514 the solid on the filter was washed with EtOAc. The mixture was added to brine and extracted 515 with EtOAc for three times. The combined organic layer was dried over $\mathrm{MgSO}_{4}$, filtered, and

516 concentrated under reduced pressure. The residue was purified by chromatography in silica gel

$517(\mathrm{DCM} / \mathrm{MeOH})$, and purified again with recrystallization ( $n$-hexane/DCM) to give the desired 518 product.

519 Proton nuclear magnetic resonance $\left({ }^{1} \mathrm{H}\right.$ NMR) spectra were recorded on Bruker 520 AVANCE $500(500 \mathrm{MHz})$. In addition, ${ }^{13} \mathrm{C}\left\{{ }^{1} \mathrm{H}\right\}$ NMR was measured on the same machine (125 $521 \mathrm{MHz}$ ), and the spectra was fully decoupled with proton by broad band decoupling. Chemical 522 shifts for NMR were quoted in parts per million ( $\mathrm{ppm}$ ) referenced to the appropriate solvent peak 523 (DMSO in DMSO- $d_{6}$ ). The abbreviation codes were adopted to describe ${ }^{1} \mathrm{H}$ NMR peak patterns; $524 \mathrm{~d}=$ doublet, $\mathrm{t}=$ triplet, and $\mathrm{br}=$ broad. Coupling constants, $J$, were displayed in Hertz unit $(\mathrm{Hz})$. 525 Infrared (IR) spectra were recorded on Bruker Alpha FT-IR spectrometer. High-resolution mass 526 spectra (HR-MS) were acquired on a high-resolution Q-TOF mass spectrometer (ionization 527 mode: ESI). 


\section{Figure Legends}

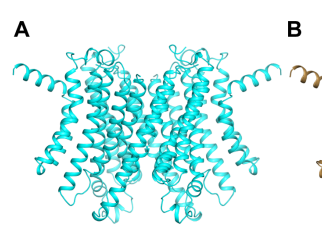

B
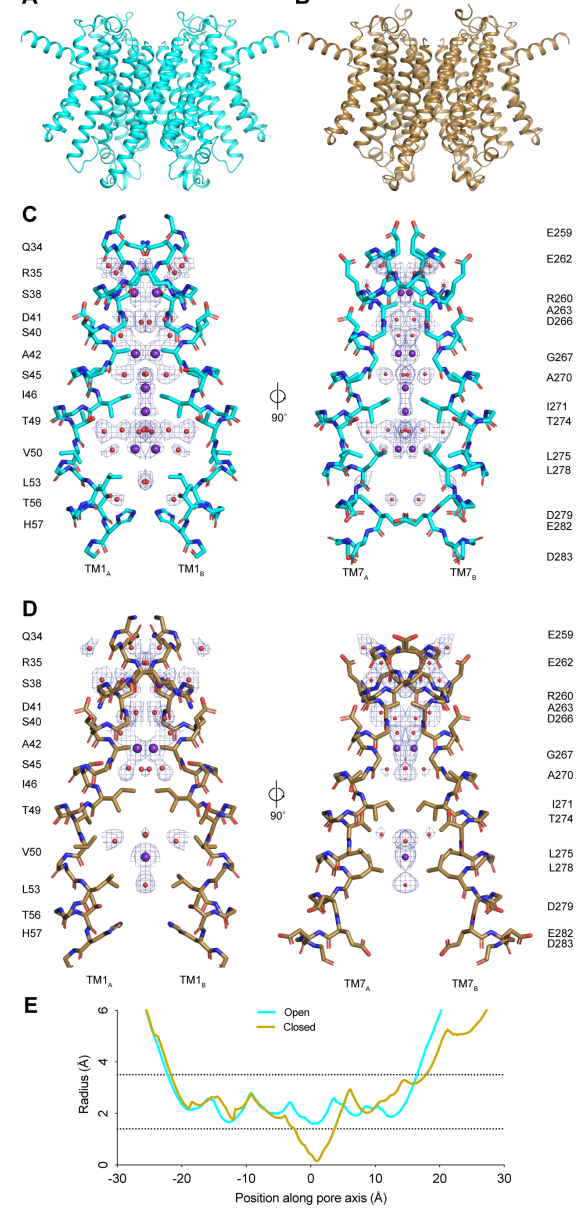

Figure 1. Structures of TMEM175 in open and closed states. (A-B) Structures of the human TMEM175 in open (A) and closed (B) states. Grey bars represent approximate width of the membrane. (C-D) Ion conduction pathways in open (C) and closed (D) states. Pore-lining residues are shown as sticks. Bound $\mathrm{K}^{+}$ions and water molecules are shown as purple and red spheres, respectively. Density peaks corresponding to $\mathrm{K}^{+}$and water molecules are shown as blue mesh and contoured at $12 \sigma$. Front and rear domains are removed for clarity (E) Plot of pore radius as a function of position along the pore axis. The 0 position corresponds to the isoleucine constriction, positive values correspond to the luminal side of the pore and negative values correspond to the cytosolic side of the pore. 

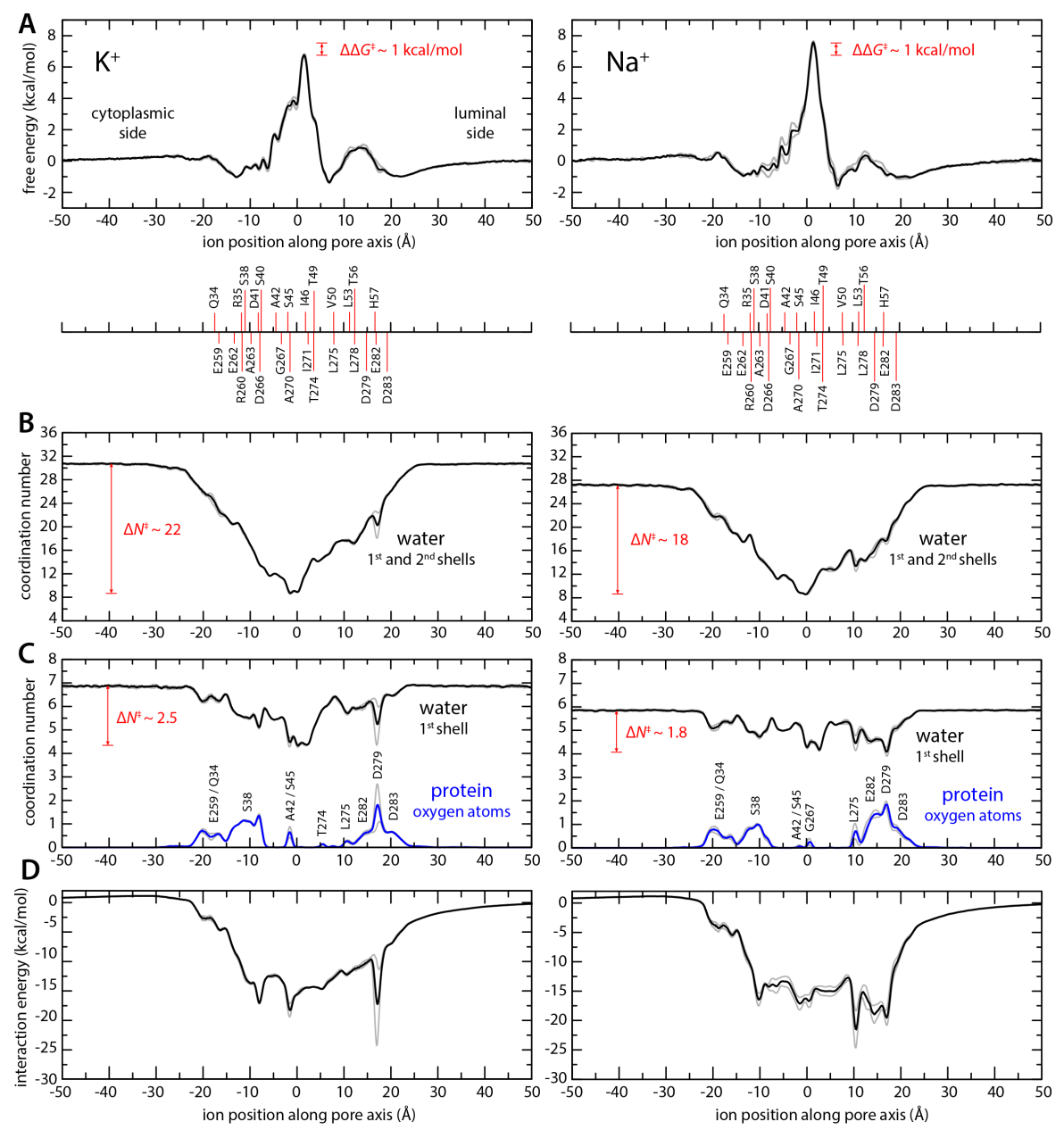

Figure 2. Enhanced-sampling MD simulations of $\mathrm{K}^{+}$and $\mathrm{Na}^{+}$permeation through

TMEM175. (A) Potential of mean force (or free energy) as a function of the ion position along the pore axis (black), for either $\mathrm{K}^{+}$(left) or $\mathrm{Na}^{+}$(right). See also Figure 2 Supplementary Figure 1. The difference between the free energy peak for $\mathrm{Na}^{+}$and that for $\mathrm{K}^{+}(\Delta \Delta G)$ is indicated. For reference, the position of selected residues in helices TM1 and TM7 is also indicated under the free-energy curves. These positions are defined as the time-average of the center-of-mass of side chain and $\mathrm{Ca}$ atoms, including equivalent protein subunits, projected along the pore axis. (B) Average number of water molecules in the first and second ion hydration shells, as a function of the ion position along the pore axis (black). The degree of depletion at the isoleucine constriction $(\Delta N)$, relative to bulk hydration, is indicated for each ion. (C) Same as (B), only for the first ion hydration shell (black). The average number of protein-oxygen atoms observed to coordinate an ion, as a function the ion position, is also shown alongside (blue), indicating the principal contributing residues. See also Figure 2 Supplementary Figure 3. (D) Ion-protein electrostatic interaction energy as a function of the ion position along the pore axis. In all panels, gray profiles represent the same quantity shown in black/blue calculated using only the first or second half of the simulation data. See Methods for further details and definitions. 

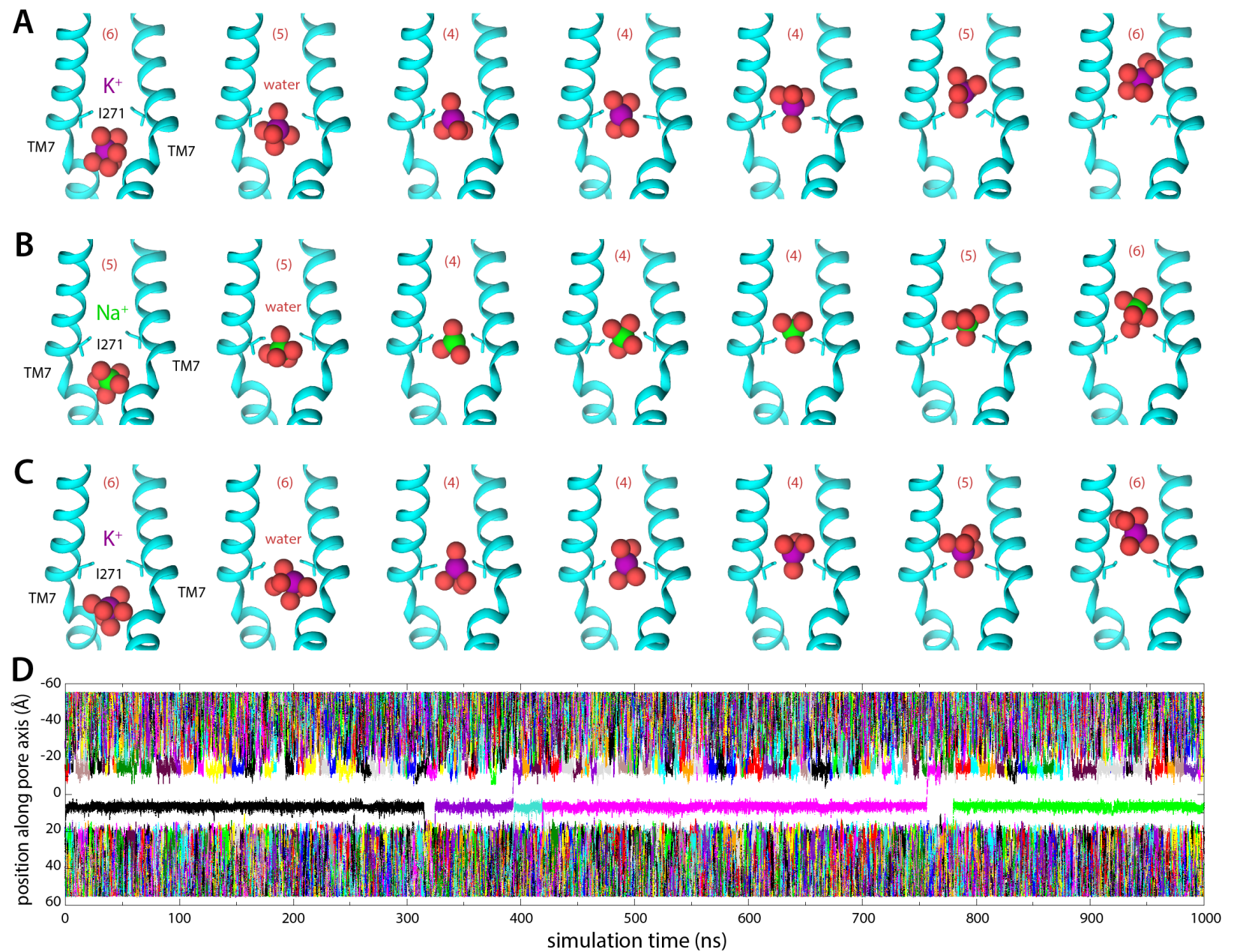

Figure 3. MD Simulations of $\mathrm{K}^{+}$and $\mathrm{Na}^{+}$permeation through hTMEM175. (A) Snapshots of

563 one of the trajectories calculated with enhanced-sampling $\mathrm{MD}$, at $0 \mathrm{mV}$ and $100 \mathrm{mM} \mathrm{KCl}$,

564 showing a $\mathrm{K}^{+}$permeation event across the isoleucine constriction. For clarity only TM7 is

565 shown, in cartoon representation, alongside the side chain of I271. Red spheres represent the

566 oxygen atoms of the water molecules in the first ion hydration shell, defined as in Figure 2. (B)

567 Same as (A), for a $\mathrm{Na}^{+}$permeation event. (C) Same as (A), from a trajectory calculated with

568 conventional MD sampling, but at $500 \mathrm{mV}$ and $400 \mathrm{mM} \mathrm{KCl}$. (D) For the latter trajectory, time

569 series of the position along the channel axis of the subset of the $\mathrm{K}^{+}$ions in the simulation found

570 within $10 \AA$ of that axis. The trajectory revealed 2 permeation events in $1 \mu \mathrm{s}$. 

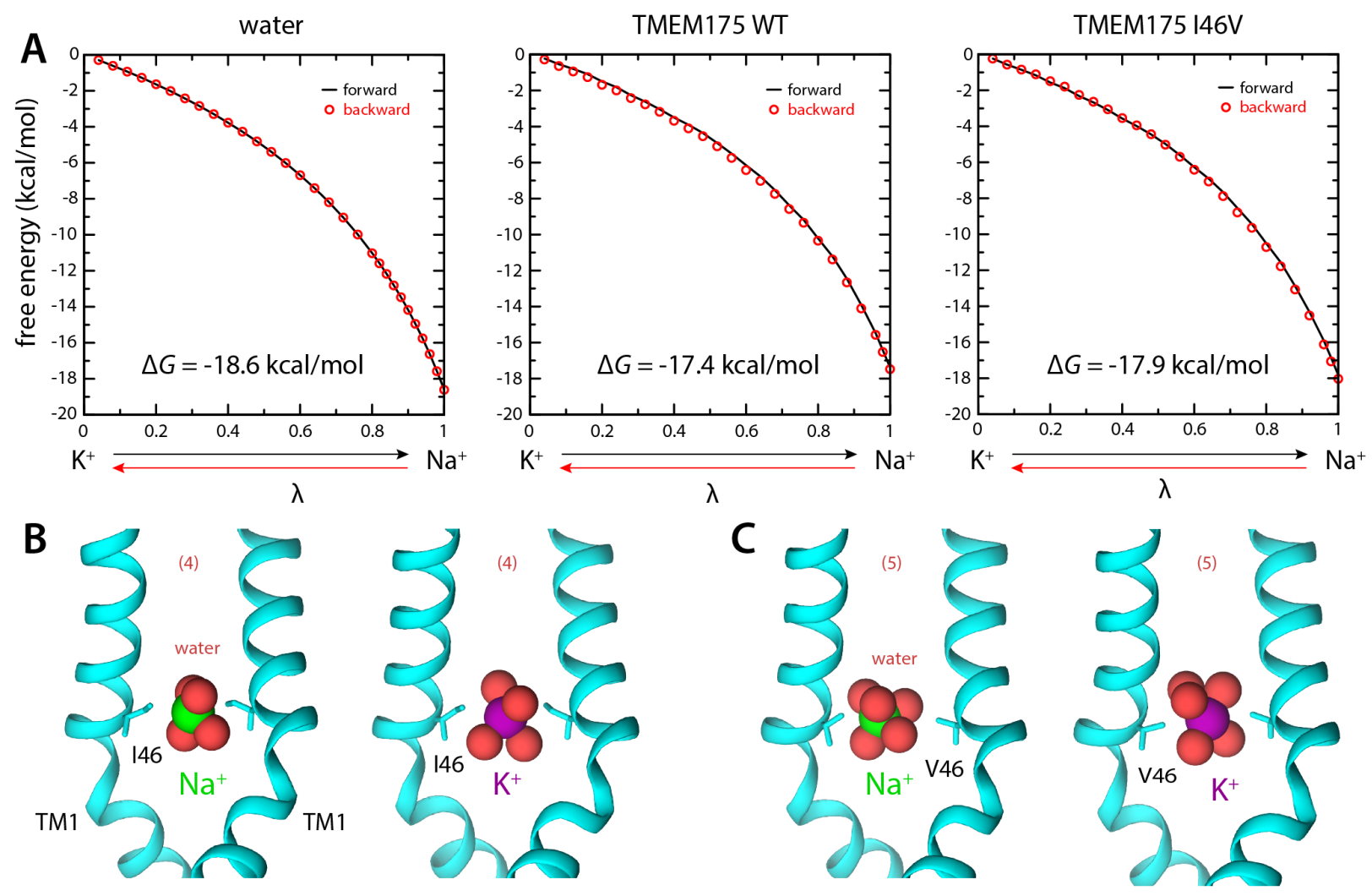

Figure 4. Ion selectivity of the isoleucine constriction. (A) Left, free-energy difference between hydrated $\mathrm{K}^{+}$and $\mathrm{Na}^{+}$, in bulk water, calculated with MD simulations using the FEP method. The free energy is plotted as a function of a varying parameter $\lambda$ that determines the size of ion $\left(\lambda=0\right.$ for $\mathrm{K}^{+}$and $\lambda=1$ for $\left.\mathrm{Na}^{+}\right)$. Results are shown for a calculation wherein $\mathrm{K}^{+}$is transformed into $\mathrm{Na}^{+}$(forward, black line) and for another carried out in the opposite direction (backward, red circles). Middle, analogous free-energy differences, calculated for an ion that is confined within the isoleucine constriction of wild-type TMEM175. Right, same as above, for the I46V mutant. (B) Snapshots of the MD/FEP simulations at the end of the forward and backward transformations, for wild-type TMEM175 and I46V mutant. For clarity only TM1 is shown, in cartoon representation, alongside the side chain of I46/V46. Red spheres represent the oxygen atoms of the water molecules in the first ion hydration shell, defined as in Figure 2. 


\section{A TMEM175}

$\mathrm{Cs}^{+}{ }_{\text {in }} / \mathrm{Na}^{+}{ }_{\text {ext }}$
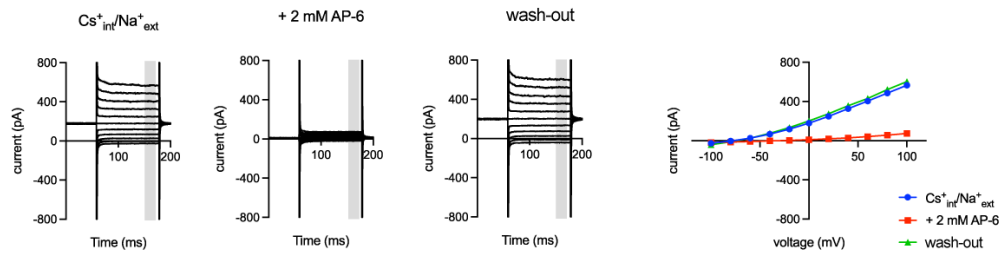

B $146 \mathrm{~V}$
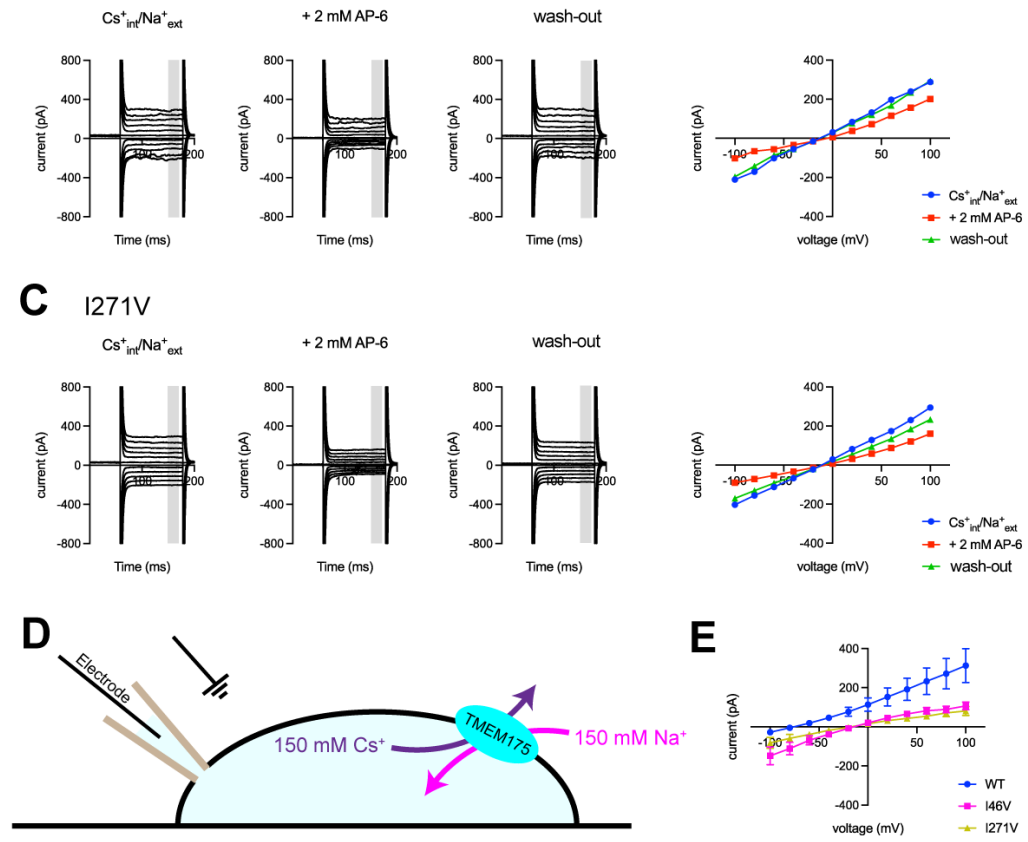

589 Representative whole-cell electrical recordings and current-voltage relationships of hTMEM175transfected (A), I46V-transfected (B), and I271V-transfected (C) HEK293T cells in the absence and presence of inhibitor AP-6, in bi-ionic conditions of $150 \mathrm{mM} \mathrm{Cs}^{+}$(intracellular) and $150 \mathrm{mM}$ $\mathrm{Na}^{+}$(extracellular). Current amplitudes in the current-voltage relationship plots were gained by averaging current values in the shaded time window. (D) A schematic of whole-cell patch clamp performed in (A-C and $\mathbf{E})(\mathbf{E})$ Current-voltage relationships of six independent whole-cell patch clamp recordings of hTMEM175 WT-, I46V-, and I271V-transfected cells in bi-ionic conditions of $150 \mathrm{mM} \mathrm{Cs}^{+}$(intracellular) and $150 \mathrm{mM} \mathrm{Na}^{+}$(extracellular). 


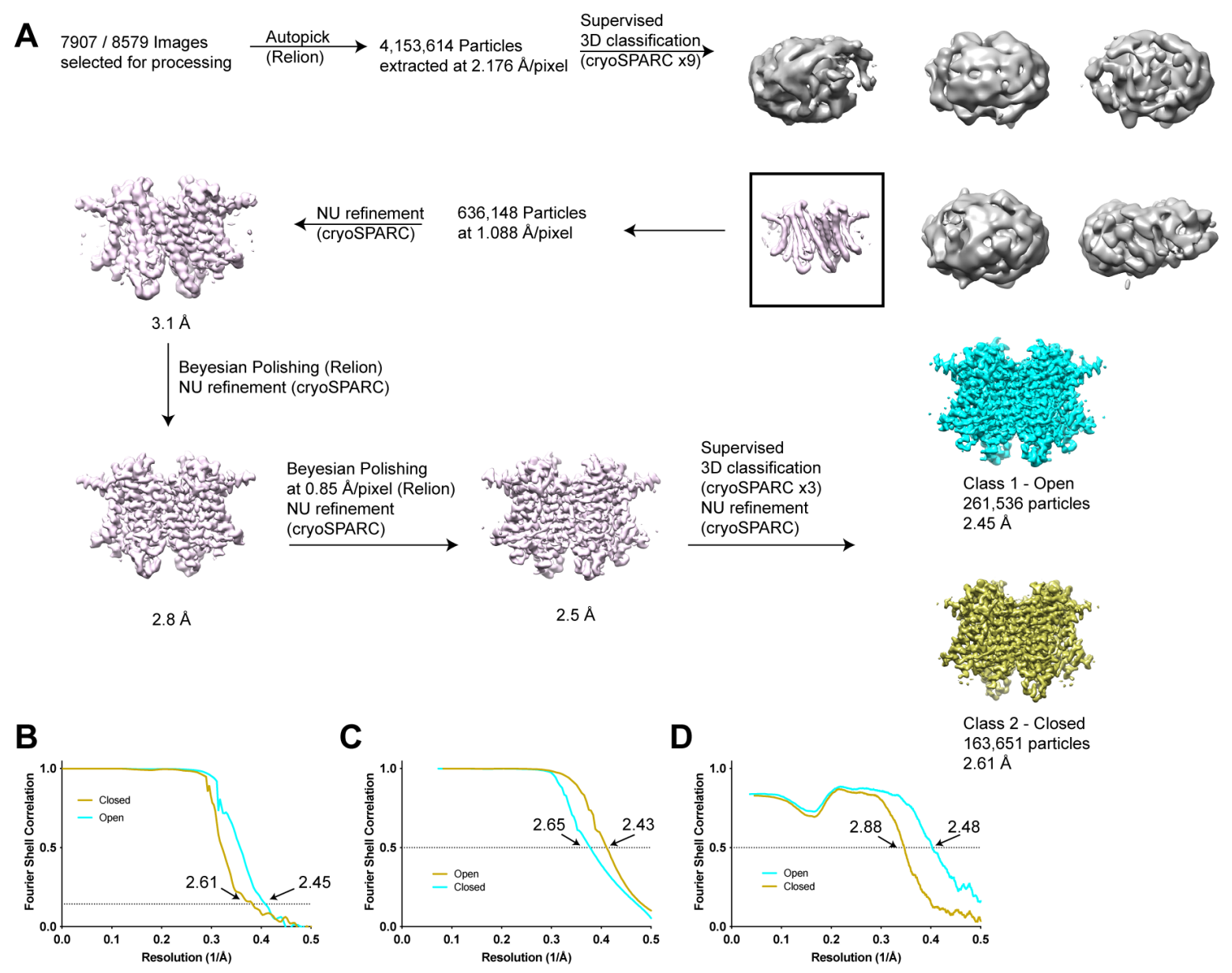

Figure 1 Supplementary Figure 1. Structure determination of human TMEM175 in open maps calculated from particles classified as open (cyan) and closed (yellow). (C) Plot of Fourier

603 shell correlations between two density-modified half-maps calculated from particles classified as 604 open (cyan) and closed (yellow). (D) Plot of Fourier shell correlations between the refined open 605 state model and density modified map of the open state (cyan) and between the refined closed 606 state model and density modified map of the closed state (yellow). 
A
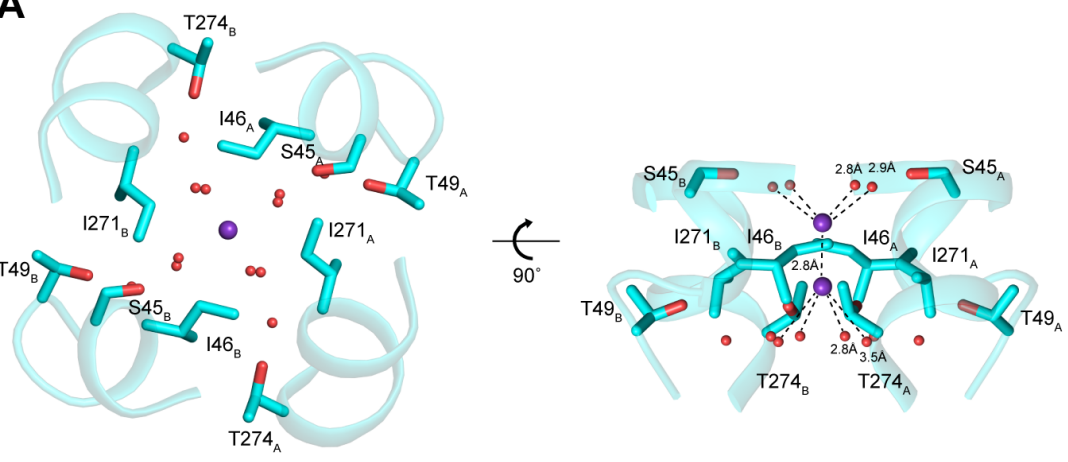

B
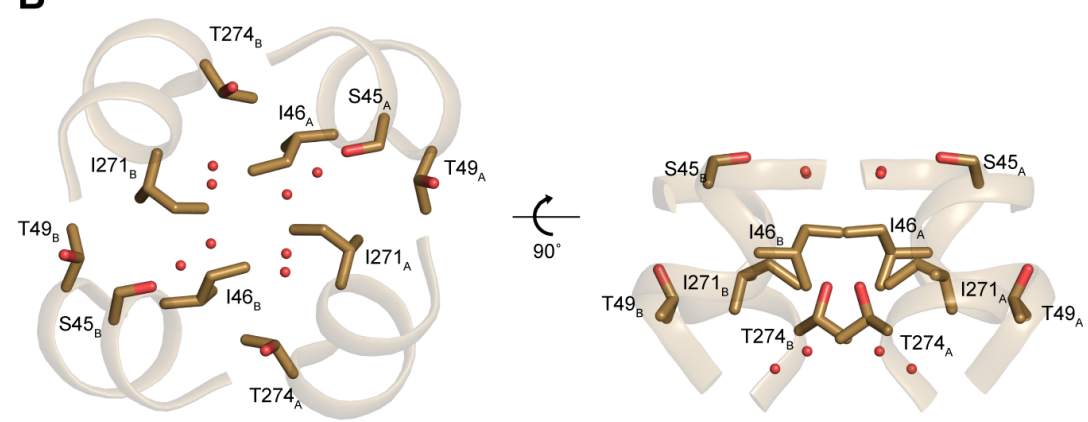

610 Figure 1 Supplementary Figure 2. Structures of the isoleucine constriction sites in open and

611 closed TMEM175 (A-B) Two views of the isoleucine constriction in open (A) and closed (B)

612 TMEM175. Ile46, Ile271 and residues that coordinate water molecules are shown as sticks.

613 Bound $\mathrm{K}^{+}$and waters are shown as purple and red spheres, respectively. 
A
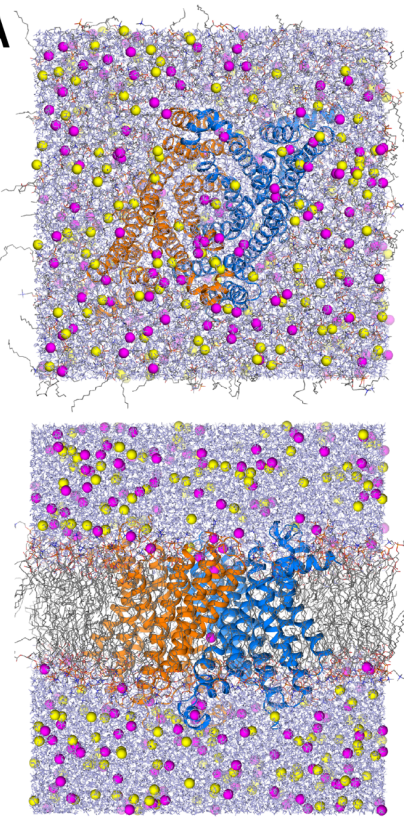

616

617

618

619

620

621

622

623

624

625
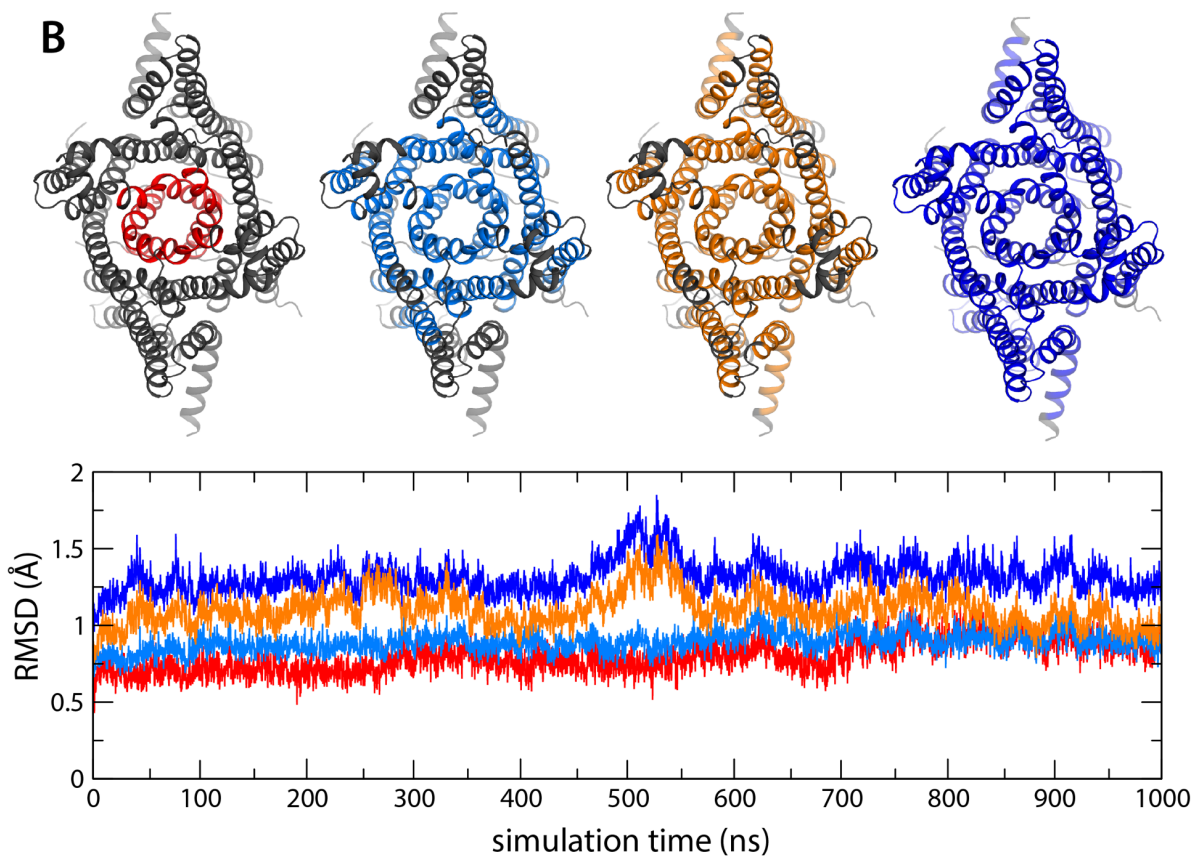

Figure 2 Supplementary Figure 1. All-atom MD simulations of hTMEM175. (A) Two views of the periodic box enclosing the simulation system, at the end of a $1-\mu$ s trajectory at $500 \mathrm{mV}$. The channel (marine/orange cartoons) is immersed in a POPC lipid bilayer (colored lines) and a $400 \mathrm{mM} \mathrm{KCl}$ buffer ( $\mathrm{K}^{+}$in magenta, $\mathrm{Cl}^{-}$in yellow, water in purple). (B) Evaluation of the deviation of the protein structure from the cryo-EM model as a function of simulation time, for the same $1-\mu$ s trajectory at $500 \mathrm{mV}$. The plot quantifies the RSMD of different portions of the channel structure, represented graphically above the plot, after least-squares self-fitting. The color code of the time-series corresponds to that used in the graphical representations. 

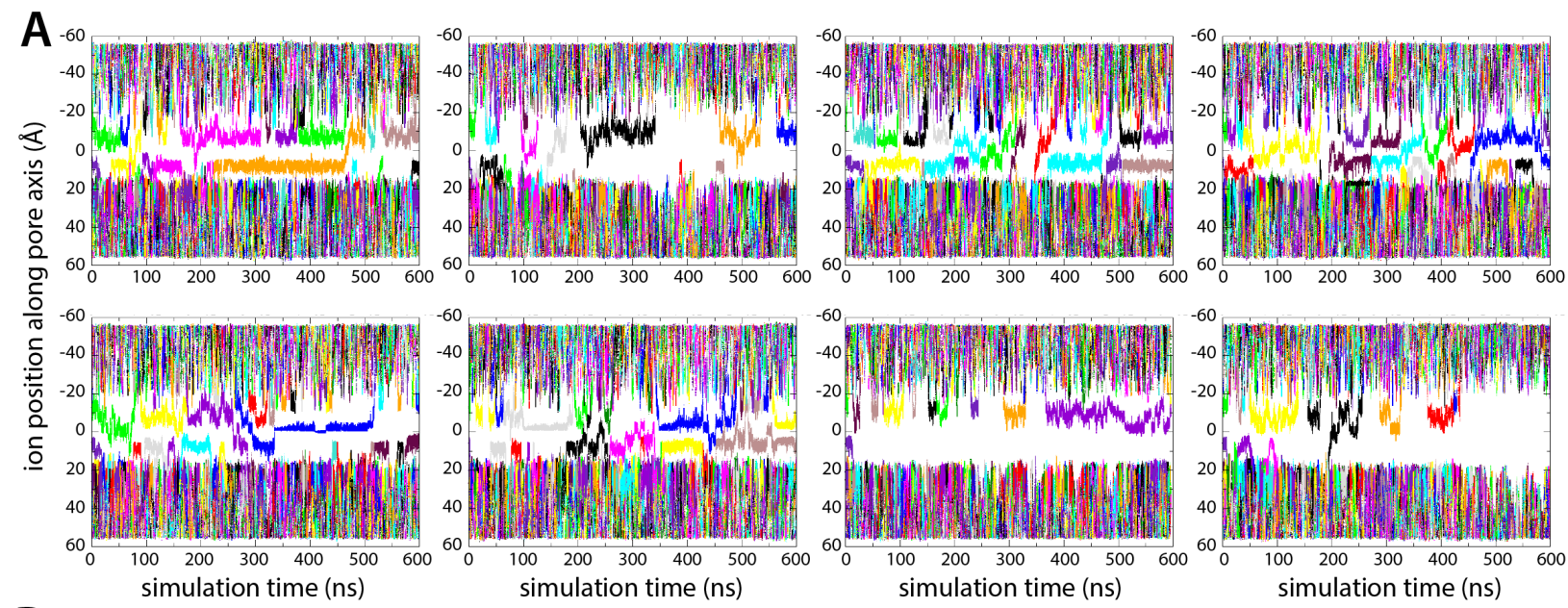

\section{B}
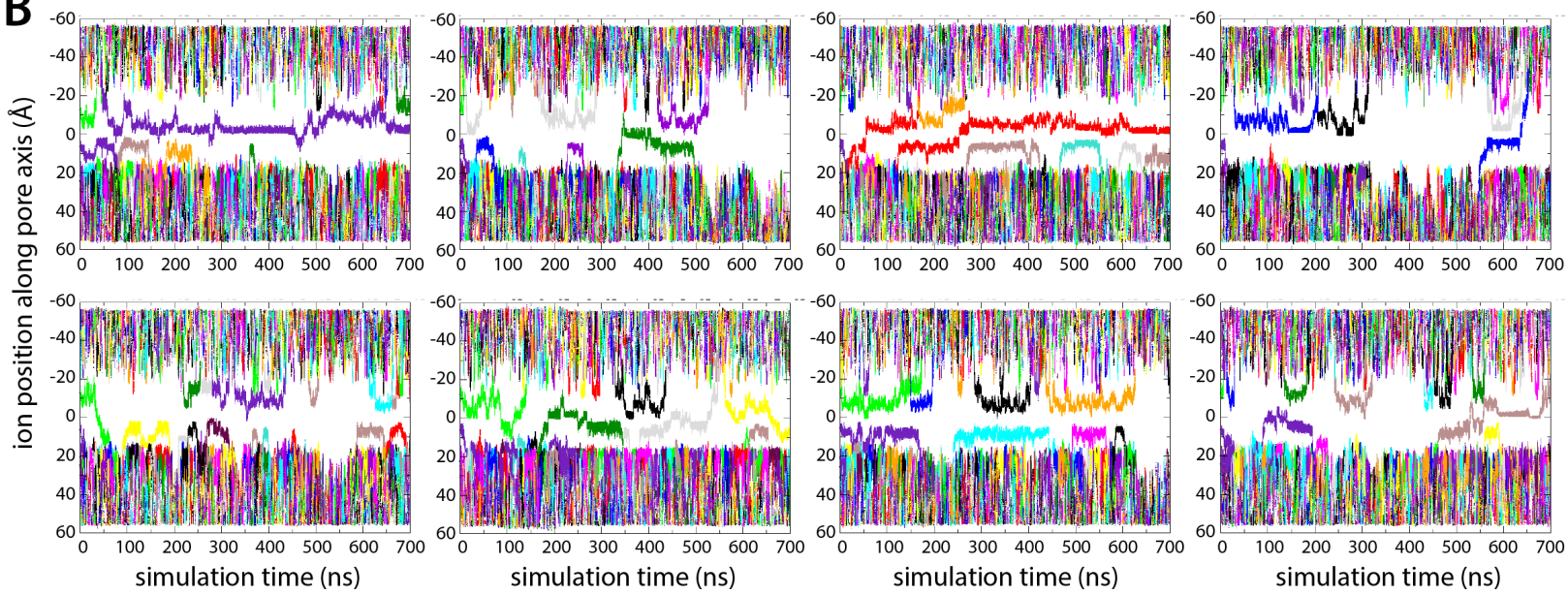

Figure 2 Supplementary Figure 2. Enhanced-sampling MD simulations of $\mathrm{K}^{+}$and $\mathrm{Na}^{+}$ permeation through hTMEM175. (A) For each of the Metadyamics trajectories calculated to examine the permeation of $\mathrm{K}^{+}$(at $0 \mathrm{mV}$ and $100 \mathrm{mM} \mathrm{KCl}$ ), time series of the position along the channel axis of the subset of the ions in the simulation found within $10 \AA$ of that axis. See also Figure 2. (B) Same as (A), for the Metadynamics trajectories calculated to study $\mathrm{Na}^{+}$permeation. 

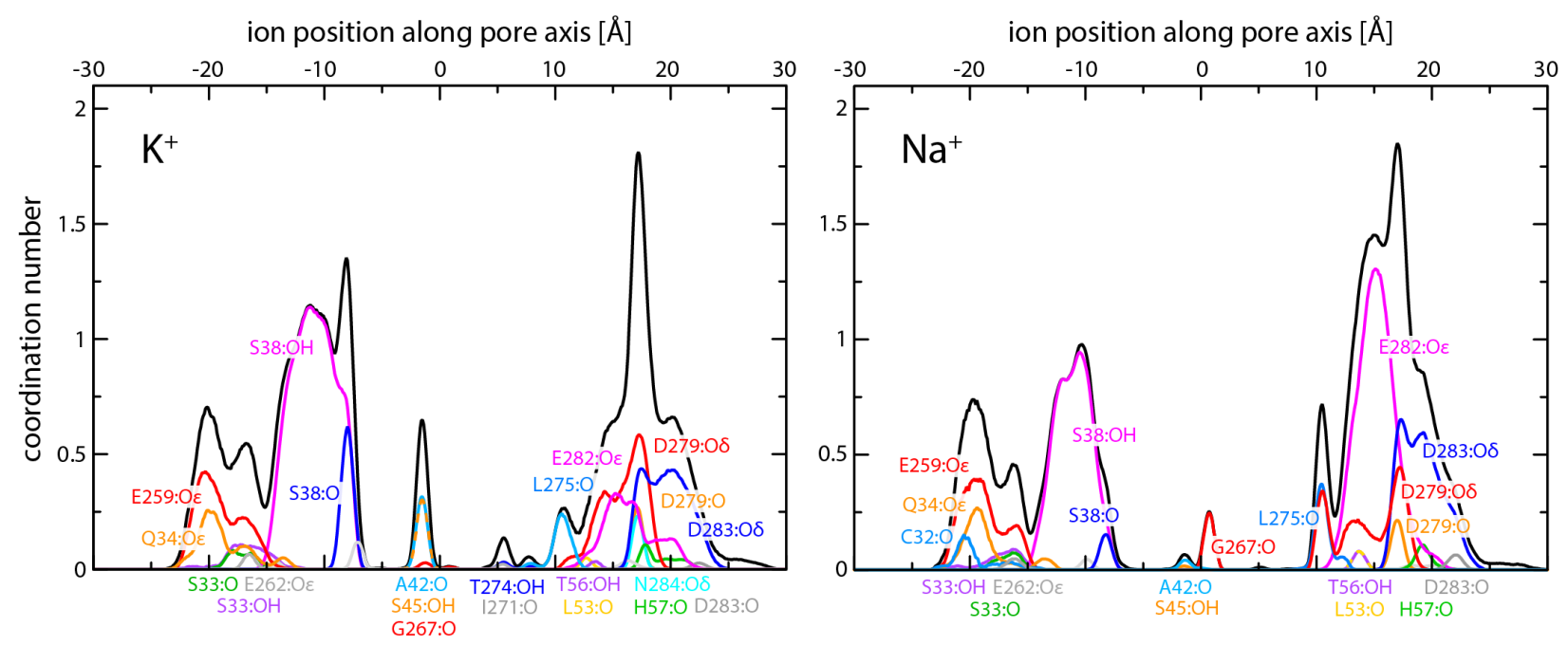

Figure 2 Supplementary Figure 3. Enhanced-sampling MD simulations of $\mathrm{K}^{+}$and $\mathrm{Na}^{+}$

637 permeation through hTMEM175. The plots report on the occurrence and persistence of direct contacts between ions and protein oxygen atoms along the central pore, as a function of the ion position along the pore axis (colors). The data reflect time-averages over the simulated trajectories. The most significant contacts are identified. The cumulative coordination number at any given position is shown alongside (black; same as in Figure 2C). 
A

644

645

646

647

648

649

B

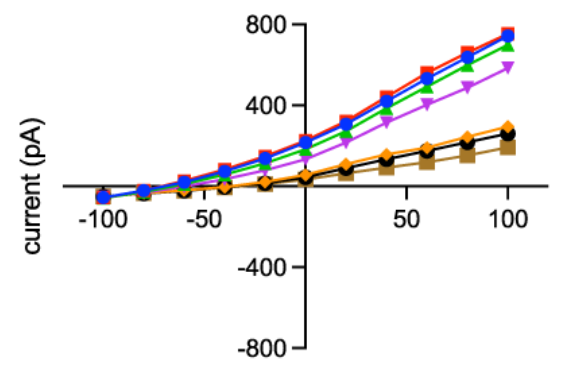

voltage $(\mathrm{mV})$

$$
\begin{aligned}
& -0 \mathrm{mM} \\
& -0.01 \mathrm{mM} \\
& -0.03 \mathrm{mM} \\
& \rightarrow 0.1 \mathrm{mM} \\
& \rightarrow 0.3 \mathrm{mM} \\
& \rightarrow 1 \mathrm{mM} \\
& --3 \mathrm{mM}
\end{aligned}
$$

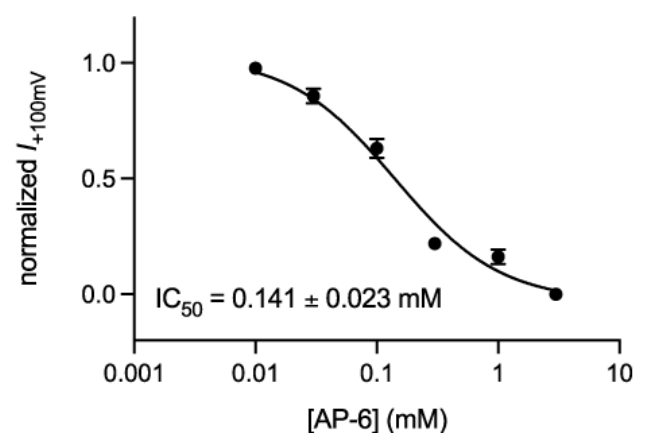

Figure 5 Supplementary Figure 1. Characterization of TMEM175 inhibition by AP-6. (A) current-voltage relationships of whole-cell patch clamp recordings of hTMEM175-transfected cells under various concentrations of AP-6. (B) concentration-response curve of Cs + current at $+100 \mathrm{mV}$ from three independent titrations. Means and S.E.M are shown. 
bioRxiv preprint doi: https://doi.org/10.1101/2021.11.05.467414; this version posted November 6, 2021. The copyright holder for this preprint (which was not certified by peer review) is the author/funder. This article is a US Government work. It is not subject to copyright under 17 USC 105 and is also made available for use under a CCO license.

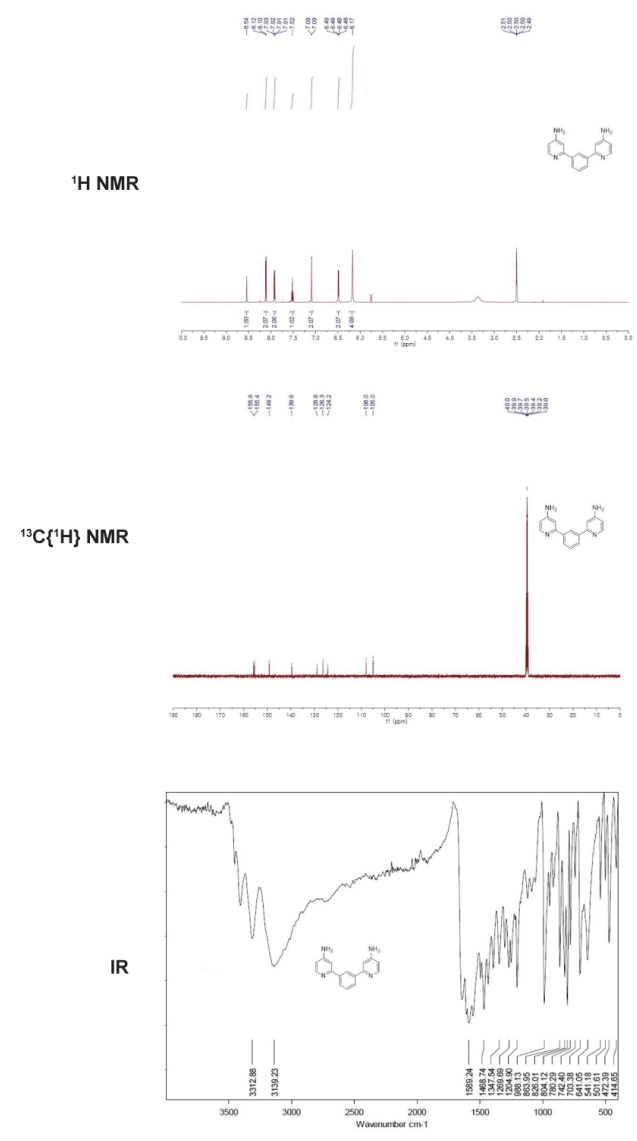

652 Figure 5 Supplementary Figure 2. Spectroscopy of AP-6. ${ }^{1} \mathrm{H}-\mathrm{NMR},{ }^{13} \mathrm{C}\left\{{ }^{1} \mathrm{H}\right\}-\mathrm{NMR}$, and IR 653 Spectrum of AP-6.

654 
4-AP: raw $\mathrm{Cs}^{+}$current at $+100 \mathrm{mV}$

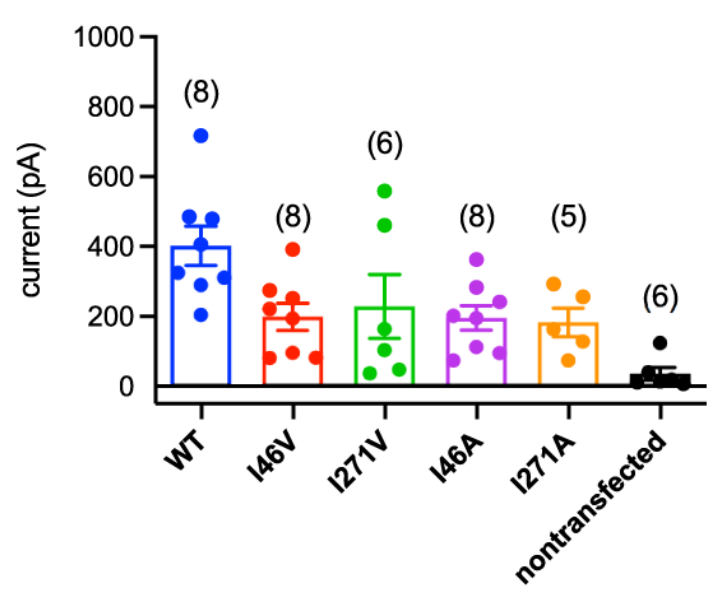

4-AP sensitive $\mathrm{Cs}^{+}$current at $+100 \mathrm{mV}$

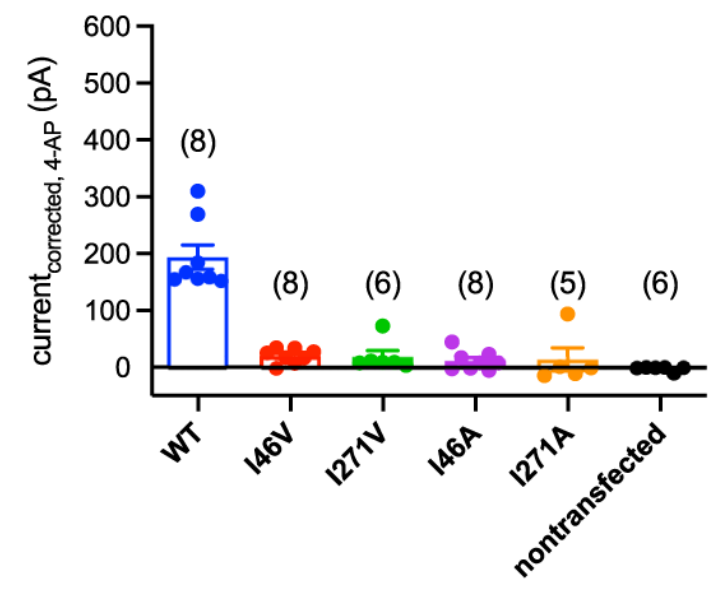

AP-6: raw $\mathrm{Cs}^{+}$current at $+100 \mathrm{mV}$

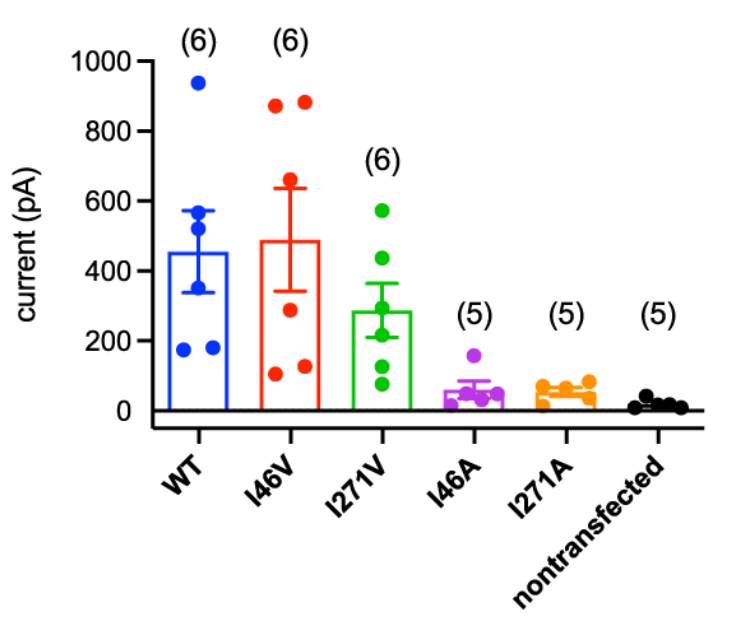

AP-6 sensitive $\mathrm{Cs}^{+}$current at $+100 \mathrm{mV}$

(6)

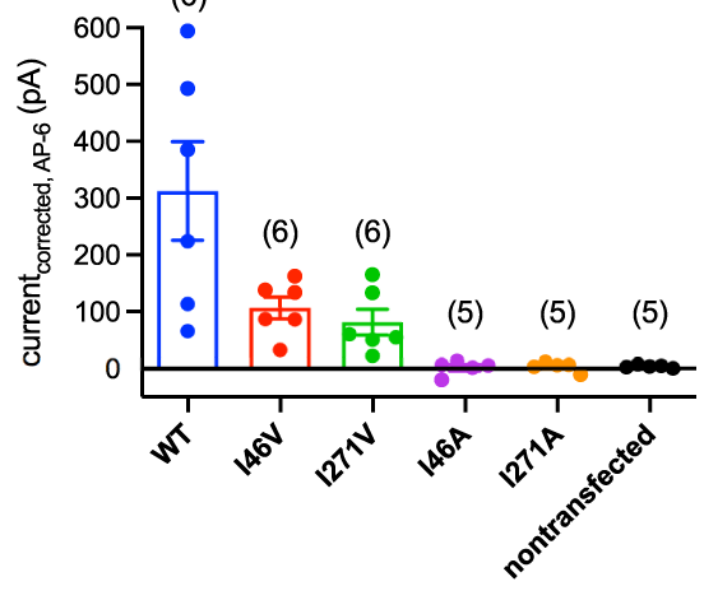

655

656

657

658

659

660

661

662

663
Figure 5 Supplementary Figure 3. 4-AP and AP-6 sensitivity of TMEM175 mutants. Mean currents recorded from HEK293T cells transfected with hTMEM175 (blue), I46V (red), I271V (green), I46A (purple), I271A (orange) and non-transfected (black) at $+100 \mathrm{mV}$ : raw currents before applying $1 \mathrm{mM}$ 4-AP (upper left), 4-AP sensitive current (bottom left), raw currents before applying $2 \mathrm{mM}$ AP-6 (upper right) and AP-6 sensitive current (bottom right). Number of experiments for each mutant are presented in parentheses. 
664

I271V<smiles>[Mg][Mg][Mg]</smiles>

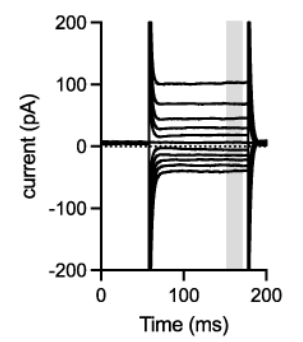

+1 mM 4-AP

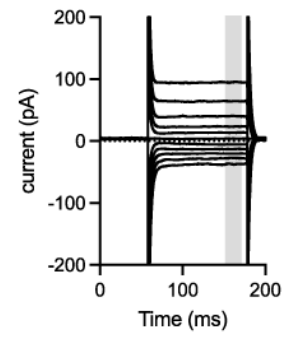

wash-out

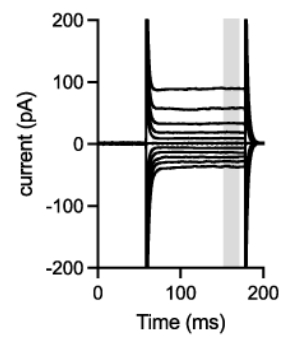

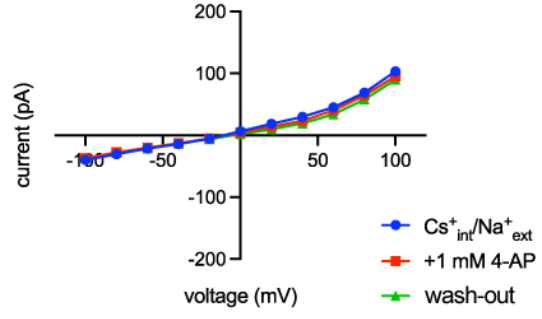

665

666 Figure 5 Supplementary Figure 4. 4-AP insensitivity of I271V mutant. 4-AP insensitivity of

667 I271V mutant. Representative whole-cell electrical recordings and current-voltage relationships

668 of I271V-transfected HEK293T cells in the absence of presence of 4-AP in bi-ionic conditions of

$669150 \mathrm{mM} \mathrm{Cs}^{+}$(intracellular) and $150 \mathrm{mM} \mathrm{Na}^{+}$(extracellular). Current amplitudes in the current-

670 voltage relationship plots were gained by averaging current values in the shaded time window.

671

672 


\section{Acknowledgements}

674 We thank the MSKCC HPC group for assistance with data processing and the members of the

675 labs for comments on the manuscript. We also thank Dr. Rahul Banerjee for early simulation

676 studies of closed-state TMEM175 not included in this manuscript. This work was supported in

677 part by a NIH-NCI Cancer Center Support Grant (P30 CA008748), the Josie Robertson

678 Investigators Program (to R.K.H.) and the Searle Scholars Program (to R.K.H), the NRF Global

679 Ph.D. Fellowship program funded by the Republic of Korea Ministry of Education

680 (2019H1A2A1076014 to J.L.) and the Division of Intramural Research of NHLBI-NIH

681 (J.D.F.G.). Computational resources were in part provided by the NIH supercomputing center

682 (Biowulf).

683 Author contributions

684 S.O. and R.K.H. designed, performed, and analyzed the cryo-EM and electrophysiological

685 experiments. W.Z., F.M. and J.D.F.G. designed, performed, and analyzed the MD simulations.

686 J.L, H.J.C. and M.K. performed and validated the chemical synthesis. S.O., W.Z., F.M., J.D.F.G.

687 and R.K.H prepared the manuscript with input from all authors.

688 Competing interests

689 The authors declare no competing interests.

690 Data and materials availability.

691 Cryo-EM maps and atomic coordinates have been deposited with the EMDB and PDB under

692 accession codes EMDB-XXXXX and PDB XXXX for open TMEM175 and codes EMDB-

693 XXXXX and PDB XXXX for closed TMEM175. All other reagents are available from the

694 corresponding author upon reasonable request.

695

696 
bioRxiv preprint doi: https://doi.org/10.1101/2021.11.05.467414; this version posted November 6, 2021. The copyright holder for this preprint (which was not certified by peer review) is the author/funder. This article is a US Government work. It is not subject to copyright under 17 USC 105 and is also made available for use under a CCO license. 


\section{References}

Adams, P.D., Afonine, P.V., Bunkoczi, G., Chen, V.B., Davis, I.W., Echols, N., Headd, J.J., Hung, L.W., Kapral, G.J., Grosse-Kunstleve, R.W., et al. (2010). PHENIX: a comprehensive Python-based system for macromolecular structure solution. Acta Crystallogr D Biol Crystallogr 66, 213-221. Allen, T.W., Andersen, O.S., and Roux, B. (2006). Molecular dynamics - potential of mean force calculations as a tool for understanding ion permeation and selectivity in narrow channels. Biophysical Chemistry 124, 251-267.

Best, R.B., Zhu, X., Shim, J., Lopes, P.E.M., Mittal, J., Feig, M., and MacKerell, A.D. (2012). Optimization of the Additive CHARMM All-Atom Protein Force Field Targeting Improved Sampling of the Backbone $\varphi, \psi$ and Side-Chain $\chi 1$ and $\chi 2$ Dihedral Angles. Journal of Chemical Theory and Computation 8, 3257-3273.

Blauwendraat, C., Heilbron, K., Vallerga, C.L., Bandres-Ciga, S., von Coelln, R., Pihlstrøm, L., Simón-Sánchez, J., Schulte, C., Sharma, M., Krohn, L., et al. (2019). Parkinson's disease age at onset genome-wide association study: Defining heritability, genetic loci, and $\alpha$-synuclein mechanisms. Movement Disorders 34, 866-875.

Bonomi, M., Branduardi, D., Bussi, G., Camilloni, C., Provasi, D., Raiteri, P., Donadio, D., Marinelli, F., Pietrucci, F., Broglia, R.A., et al. (2009). PLUMED: A portable plugin for free-energy calculations with molecular dynamics. Computer Physics Communications 180, 1961-1972.

Brooks, B.R., Brooks lii, C.L., Mackerell Jr, A.D., Nilsson, L., Petrella, R.J., Roux, B., Won, Y., Archontis, G., Bartels, C., Boresch, S., et al. (2009). CHARMM: The biomolecular simulation program. Journal of Computational Chemistry 30, 1545-1614.

Cang, C., Aranda, K., Seo, Y.J., Gasnier, B., and Ren, D. (2015). TMEM175 Is an Organelle K(+) Channel Regulating Lysosomal Function. Cell 162, 1101-1112.

Crespo, Y., Marinelli, F., Pietrucci, F., and Laio, A. (2010). Metadynamics convergence law in a multidimensional system. Physical Review E 81, 055701.

Doyle Declan, A., Cabral João, M., Pfuetzner Richard, A., Kuo, A., Gulbis Jacqueline, M., Cohen Steven, L., Chait Brian, T., and MacKinnon, R. (1998). The Structure of the Potassium Channel: Molecular Basis of K+ Conduction and Selectivity. Science 280, 69-77.

Emsley, P., Lohkamp, B., Scott, W.G., and Cowtan, K. (2010). Features and development of Coot. Acta Crystallogr D Biol Crystallogr 66, 486-501.

Iwaki, H., Blauwendraat, C., Leonard, H.L., Liu, G., Maple-Grodem, J., Corvol, J.C., Pihlstrom, L., van Nimwegen, M., Hutten, S.J., Nguyen, K.H., et al. (2019). Genetic risk of Parkinson disease and progression:: An analysis of 13 longitudinal cohorts. Neurol Genet 5, e348.

Jinn, S., Blauwendraat, C., Toolan, D., Gretzula, C.A., Drolet, R.E., Smith, S., Nalls, M.A., Marcus, J., Singleton, A.B., and Stone, D.J. (2019). Functionalization of the TMEM175 p.M393T Variant as a risk factor for Parkinson Disease. Hum Mol Genet.

Jinn, S., Drolet, R.E., Cramer, P.E., Wong, A.H., Toolan, D.M., Gretzula, C.A., Voleti, B., Vassileva, G., Disa, J., Tadin-Strapps, M., et al. (2017). TMEM175 deficiency impairs lysosomal and mitochondrial function and increases alpha-synuclein aggregation. Proc Natl Acad Sci U S A 114, 2389-2394.

Klauda, J.B., Venable, R.M., Freites, J.A., O’Connor, J.W., Tobias, D.J., Mondragon-Ramirez, C., Vorobyov, I., MacKerell, A.D., and Pastor, R.W. (2010). Update of the CHARMM All-Atom Additive 
Force Field for Lipids: Validation on Six Lipid Types. The Journal of Physical Chemistry B 114, 78307843.

Lee, C., Guo, J., Zeng, W., Kim, S., She, J., Cang, C., Ren, D., and Jiang, Y. (2017). The lysosomal potassium channel TMEM175 adopts a novel tetrameric architecture. Nature 547, 472-475. Long, S.B., Campbell, E.B., and Mackinnon, R. (2005). Crystal structure of a mammalian voltagedependent Shaker family K+ channel. Science 309, 897-903.

Marinelli, F., Pietrucci, F., Laio, A., and Piana, S. (2009). A Kinetic Model of Trp-Cage Folding from Multiple Biased Molecular Dynamics Simulations. PLOS Computational Biology 5, e1000452. Mironenko, A., Zachariae, U., de Groot, B.L., and Kopec, W. (2021). The Persistent Question of Potassium Channel Permeation Mechanisms. Journal of Molecular Biology 433, 167002.

Nalls, M.A., Pankratz, N., Lill, C.M., Do, C.B., Hernandez, D.G., Saad, M., DeStefano, A.L., Kara, E., Bras, J., Sharma, M., et al. (2014). Large-scale meta-analysis of genome-wide association data identifies six new risk loci for Parkinson\&\#39;s disease. Nature Genetics 46, 989.

Nina, M., Beglov, D., and Roux, B. (1997). Atomic Radii for Continuum Electrostatics Calculations Based on Molecular Dynamics Free Energy Simulations. The Journal of Physical Chemistry B 101, 5239-5248.

Oh, S., Paknejad, N., and Hite, R.K. (2020). Gating and selectivity mechanisms for the lysosomal $\mathrm{K}(+)$ channel TMEM175. Elife 9.

Pastor, R.W., and MacKerell, A.D. (2011). Development of the CHARMM Force Field for Lipids. The Journal of Physical Chemistry Letters 2, 1526-1532.

Phillips, J.C., Braun, R., Wang, W., Gumbart, J., Tajkhorshid, E., Villa, E., Chipot, C., Skeel, R.D., Kalé, L., and Schulten, K. (2005). Scalable molecular dynamics with NAMD. Journal of Computational Chemistry 26, 1781-1802.

Prakriya, M., Feske, S., Gwack, Y., Srikanth, S., Rao, A., and Hogan, P.G. (2006). Orai1 is an essential pore subunit of the CRAC channel. Nature 443, 230-233.

Punjani, A., and Fleet, D.J. (2021). 3D variability analysis: Resolving continuous flexibility and discrete heterogeneity from single particle cryo-EM. Journal of Structural Biology 213, 107702.

Punjani, A., Rubinstein, J.L., Fleet, D.J., and Brubaker, M.A. (2017). cryoSPARC: algorithms for rapid unsupervised cryo-EM structure determination. Nature Methods 14, 290-296.

Punjani, A., Zhang, H., and Fleet, D.J. (2020). Non-uniform refinement: adaptive regularization improves single-particle cryo-EM reconstruction. Nature Methods 17, 1214-1221.

Raiteri, P., Laio, A., Gervasio, F.L., Micheletti, C., and Parrinello, M. (2006). Efficient Reconstruction of Complex Free Energy Landscapes by Multiple Walkers Metadynamics. The Journal of Physical Chemistry B 110, 3533-3539.

Rohou, A., and Grigorieff, N. (2015). CTFFIND4: Fast and accurate defocus estimation from electron micrographs. J Struct Biol 192, 216-221.

Roux, B. (2008). The Membrane Potential and its Representation by a Constant Electric Field in Computer Simulations. Biophysical Journal 95, 4205-4216.

Staritzbichler, R., Anselmi, C., Forrest, L.R., and Faraldo-Gómez, J.D. (2011). GRIFFIN: A Versatile Methodology for Optimization of Protein-Lipid Interfaces for Membrane Protein Simulations. Journal of Chemical Theory and Computation 7, 1167-1176.

Tao, X., Hite, R.K., and MacKinnon, R. (2017). Cryo-EM structure of the open high-conductance Ca2+-activated K+ channel. Nature 541, 46-51. 
Terwilliger, T.C., Ludtke, S.J., Read, R.J., Adams, P.D., and Afonine, P.V. (2019). Improvement of cryo-EM maps by density modification. bioRxiv, 845032.

785 Wang, W., Zhang, X., Gao, Q., Lawas, M., Yu, L., Cheng, X., Gu, M., Sahoo, N., Li, X., Li, P., et al. 786 (2017). A voltage-dependent $\mathrm{K}+$ channel in the lysosome is required for refilling lysosomal Ca2+ stores. Journal of Cell Biology 216, 1715-1730.

788 Wie, J., Liu, Z., Song, H., Tropea, T.F., Yang, L., Wang, H., Liang, Y., Cang, C., Aranda, K., Lohmann, 789 J., et al. (2021). A growth-factor-activated lysosomal K+ channel regulates Parkinson's pathology.

$790 \quad$ Nature 591, 431-437.

791 Zheng, S.Q., Palovcak, E., Armache, J.P., Verba, K.A., Cheng, Y., and Agard, D.A. (2017). 792 MotionCor2: anisotropic correction of beam-induced motion for improved cryo-electron 793 microscopy. Nat Methods 14, 331-332.

794 Zhong, X.Z., Sun, X., Cao, Q., Dong, G., Schiffmann, R., and Dong, X.-P. (2016). BK channel agonist 795 represents a potential therapeutic approach for lysosomal storage diseases. Scientific Reports 6 , 79633684.

797 Zhou, W.C., Marinelli, F., Nief, C., and Faraldo-Gomez, J.D. (2017). Atomistic simulations indicate 798 the c-subunit ring of the F1Fo ATP synthase is not the mitochondrial permeability transition pore. 799 Elife 6.

800 Zhu, F., and Hummer, G. (2012). Theory and Simulation of Ion Conduction in the Pentameric GLIC 801 Channel. Journal of Chemical Theory and Computation 8, 3759-3768.

802 Zivanov, J., Nakane, T., Forsberg, B.O., Kimanius, D., Hagen, W.J., Lindahl, E., and Scheres, S.H. 803 (2018). New tools for automated high-resolution cryo-EM structure determination in RELION-3.

804 Elife 7.

805 Zivanov, J., Nakane, T., and Scheres, S.H.W. (2019). A Bayesian approach to beam-induced motion 806 correction in cryo-EM single-particle analysis. IUCrJ 6, 5-17. 